\title{
FOURIER SPECTRAL APPROXIMATION TO A DISSIPATIVE SYSTEM MODELING THE FLOW OF LIQUID CRYSTALS*
}

\author{
QIANG DU†, BENYU GUO , AND JIE SHEN $^{\ddagger}$
}

\begin{abstract}
We study a Fourier-spectral method for a dissipative system modeling the flow of liquid crystals. We first prove its convergence in a suitable sense and establish the existence of a global weak solution of the original problem and its uniqueness in the two dimensional case. Then we derive error estimates which exhibit the spectral accuracy of the Fourier-spectral method. We also construct a fully discrete scheme and carry out a complete stability and error analysis for it. Finally, we present some illustrative numerical results.
\end{abstract}

Key words. liquid crystals, Fourier-spectral method, convergence, error estimate

AMS subject classifications. 65N35, 65M60, 76D99

PII. S0036142900373737

1. Introduction. Liquid crystals are sometimes referred to as the fourth state of matter following gases, liquids, and solids. They provide effective solutions to various problems in scientific and industrial applications. For many problems, a liquid crystal molecule is often pictured as a rod whose orientation is depicted by a director field $d$. In many previous works, the velocity field $u$ of liquid crystal is completely ignored, while in some other works, $u$ is taken into account. Attempts to couple the states of the director with the states of flow have become available only recently (see, for example, [8]). For other recent analytical and numerical studies of nematic liquid crystals, we refer to $[5,11,25]$.

In this paper, we study a Fourier-spectral approximation for the following nonlinear system for the pair $(u, d)$ :

$$
\left\{\begin{array}{rlrl}
\frac{\partial u}{\partial t}+(u \cdot \nabla) u-\nu \nabla \cdot D(u)+\nabla p+\lambda \nabla \cdot(\nabla d \odot \nabla d)=0, & & (x, t) \in Q_{T} \\
\nabla \cdot u=0, & (x, t) \in Q_{T} \\
\frac{\partial d}{\partial t}+(u \cdot \nabla) d-\gamma(\Delta d-f(d))=0, & & (x, t) \in Q_{T}
\end{array}\right.
$$

with the initial values $u(x, 0)=u_{0}(x), d(x, 0)=d_{0}(x)$, and certain boundary conditions. Here, $\Omega \subseteq \mathcal{R}^{n}(n=2,3), Q_{T}=\omega \times(0, T], \nu, \lambda$, and $\gamma$ are some positive constants. $p$ is the pressure, $\nabla d$ is the Jacobian matrix with the $(j, k)$ th entry

*Received by the editors June 12, 2000; accepted for publication (in revised form) January 7, 2001; published electronically June 26, 2001.

http://www.siam.org/journals/sinum/39-3/37373.html

${ }^{\dagger}$ Department of Mathematics, Hong Kong University of Science and Technology, Clear Water Bay, Kowloon, Hong Kong (madu@uxmail.ust.hk). This authors's research was supported in part by a grant from the HKRGC and by Chinese key project G199903280.

${ }_{\ddagger}$ Department of Mathematics, Shanghai Normal University, Shanghai, China (byguo@guomai. sh.cn). This author's research was supported in part by Chinese key project G199903280. Part of the research was completed during the author's visit to the Hong Kong University of Science and Technology.

$\S$ Department of Mathematics, Pennsylvania State University, University Park, PA 16802 (shen@ math.psu.edu). Current address: Department of Mathematics, University of Central Florida, Orlando, FL 32816. This author's research was supported in part by NSF grants DMS-9706951 and DMS-0074283. Part of the research was completed during the author's visit to the Hong Kong University of Science and Technology. 
$\frac{\partial d^{(j)}}{\partial x_{k}}, \nabla d \odot \nabla d$ is the stretching tensor whose $(j, k)$ th entry is given by $\frac{\partial d}{\partial x_{j}} \cdot \frac{\partial d}{\partial x_{k}}$, $D(u)=\frac{1}{2}\left(\nabla u+(\nabla u)^{T}\right)$, and $\nabla \cdot D(u)$ is a vector whose $j$ th component is given by

$$
\frac{1}{2} \sum_{k=1}^{n} \frac{\partial}{\partial x_{k}}\left(\frac{\partial u^{(k)}}{\partial x_{j}}+\frac{\partial u^{(j)}}{\partial x_{k}}\right)
$$

The above system (1.1) was first proposed in Lin [19, 20]. It was motivated by the Erickson-Leslie equation describing the flow of nematic liquid crystals; see Erickson $[9,10]$ and Leslie [18]. The system is not parabolic, but it is dissipative. The system (1.1) was derived with much simplification from the original free energy functional in the Erickson-Leslie theory, but it retains all the essential mathematical features (see $[19,20])$.

Lin and Liu [22] considered the existence and uniqueness of the solution of (1.1) when $\Omega$ is bounded, $f(d)$ is bounded uniformly for all $d \in \mathcal{R}^{n}$, and the Dirichlet boundary condition is imposed. Subsequently, Liu and Walkington [24] provided a finite element approximation to (1.1), where $f(d)$ is the gradient of a scalar valued function $F(d)$, namely,

$$
F(d)=\frac{1}{4 \epsilon^{2}}\left(|d|^{2}-1\right)^{2}, \quad f(d)=\nabla_{d} F(d)=\frac{1}{\epsilon^{2}}\left(|d|^{2}-1\right) d, \quad \epsilon>0 .
$$

The penalty term $f(d)$ is used to approximate the constraint $|d|=1$. It was truncated to be quadratic outside the ball of $|d| \geq 1$ in Liu and Walkington [24].

In this paper, we assume periodic boundary conditions for both the velocity and the director. Physically speaking, there are situations where the properties of the liquid crystals are characterized by periodic patterns [15] and are studied in periodic cells [2]. Thus, periodic boundary conditions are of physical relevance in such settings. On the other hand, the defects in the center of the bulk of a liquid crystal configuration can be studied separately by ignoring the influence of the boundary conditions when the defects are far away from the boundary. In this case, the periodicity does not significantly affect the defect motion and the interaction with the flow.

With the periodic boundary conditions, the Fourier-spectral approximation becomes a very natural way to solve the model equations numerically. Indeed, with the aforementioned penalization, the underlying director and velocity fields become quite regular. Employing the Fourier-spectral methods in the approximation may allow us to take the full advantage of the high resolution nature of the spectral methods. In fact, the Fourier-spectral methods have been successfully applied to the numerical simulation of microstructural evolution using diffusive phase field models, where the thickness of the diffusive interface plays the same role as the small parameter $\epsilon$ in the penalty term; cf. [4, 31]. We must emphasize that the Fourier-spectral methods we propose here, as well as the finite element methods used in [24, 27], are not suitable for computing the singular limiting solutions as $\epsilon \rightarrow 0$; instead, it is aimed at computing the regularized solutions with small but fixed penalty parameter $\epsilon$. We refer to [1] for works related to the algorithms for the $\epsilon \rightarrow 0$ limiting case of harmonic mappings.

Thus, we consider in this paper a Fourier-spectral approximation to problem (1.1) with the periodic boundary condition on the domain $\Omega=(0,2 \pi)^{d}$. We shall construct a Fourier-spectral approximation and derive optimal error estimates of spectral-type for it. The convergence of the Fourier-spectral approximation also implies the existence of a global weak solution for the system (1.1) with periodic boundary conditions and its uniqueness in the two dimensional case. In our analysis, we do not assume 
$f(d)$ to be uniformly bounded, so no truncation on the penalty function $f$ in (1.2) is required.

Of course, the Fourier-spectral approximation has its limitation when the boundary effect becomes important for the defect motion and interaction. In cases where periodic assumption becomes inappropriate due to the boundary effects in one or more directions, one may use a Legendre- or Chebyshev-Galerkin approximation $[14,28,29]$ in these nonperiodic directions. The theoretical frameworks presented here for the periodic boundary conditions can mostly be applied to the latter case, although the actual numerical implementations will be much more involved, mainly due to the difficulty associated with the incompatibility, which, by the way, can be easily handled in the periodic case considered here. One may also consider using a finite-element or finite-difference method as in [24, 27]. In fact, finite-element and finite-difference methods have played dominant roles in the simulation of liquid crystals $[6,7,26]$. However, the difficulties involved in a finite-difference method or finite-element method can be formidable for the liquid crystal flows considered here. Through this and future works, we hope to demonstrate that spectral methods may provide a valuable alternative for conducting simulations of liquid crystal flows in certain physically relevant situations.

The paper is organized as follows. In section 2 , we prove the convergence of a sequence of numerical solutions to a global weak solution of problem (1.1). The uniqueness of such solution when $n=2$ is then considered. In section 3 , we derive an error estimate which shows the spectral accuracy of the proposed numerical method. In section 4, we take into account the discretization in time and construct some fully discrete schemes. Some illustrative numerical experiments are presented in the final section.

2. Fourier spectral method. Let all functions in (1.1) be periodic with period $2 \pi$ for all $x_{j}, 1 \leq j \leq n$. For simplicity, we use the notations $\partial_{t}=\frac{\partial}{\partial t}$ and $\partial_{x_{j}}=$ $\frac{\partial}{\partial x_{j}}, 1 \leq j \leq n$. Let $\mathcal{D}_{p}$ be the set of all infinitely differentiable, $n$-dimensional vector periodic functions with the period $2 \pi$. For $1 \leq q \leq \infty, L_{p}^{q}$ is the closure of $\mathcal{D}_{p}$ in $L^{q}(\Omega)$ with the norm $\|\cdot\|_{L^{q}}$. Similarly, for $r \geq 0, H_{p}^{r}$ stands for the closure of $\mathcal{D}_{p}$ in $H^{r}(\Omega)$, equipped with the seminorm $|\cdot|_{r}=|\cdot|_{H^{r}(\Omega)}$ and the norm $\|\cdot\|_{r}=\|\cdot\|_{H^{r}(\Omega)}$. For any $r<0, H_{p}^{r}$ is the duality of $H_{p}^{-r}$. In particular, we denote $\|\cdot\|=\|\cdot\|_{0}$. For any $v, w \in \mathcal{R}^{n}$,

$$
(v, w)=\int_{\Omega} v \cdot w d x=\int_{\Omega} v^{T} w d x
$$

We set

$$
\mathcal{D}_{p}^{*}=\left\{v \in \mathcal{D}_{p} \mid \nabla \cdot v=0\right\}
$$

and denote by $H$ and $V$ the closures of $\mathcal{D}_{p}^{*}$ in $L_{p}^{2}$ and $H_{p}^{1}$, respectively.

For any $v, w \in H_{p}^{1}$, let

$$
(D(v), D(w))=\int_{\Omega} D(v) \cdot D(w) d x .
$$

It can be checked that for any $v \in H_{p}^{2}$ and $w \in H_{p}^{1}$,

$$
(D(v), D(w))+((\nabla \cdot D v), w)=0 .
$$


Also, one can easily show (cf. [22]) that for any $v \in H_{p}^{2}$,

$$
\nabla \cdot(\nabla v \odot \nabla v)=\nabla\left(\frac{|\nabla v|^{2}}{2}\right)+(\nabla v)^{T} \Delta v .
$$

A weak formulation of the problem (1.1) with periodic boundary condition is to find a pair $(u, d) \in L^{2}(0, T ; V) \cap L^{\infty}(0, T ; H) \times L^{2}\left(0, T ; H_{p}^{2}\right) \cap L^{\infty}\left(0, T ; H_{p}^{1}\right)$ such that for any $v \in V$ and $w \in H_{p}^{2}$,

$$
\left\{\begin{aligned}
\left(\partial_{t} u, v\right)+((u \cdot \nabla) u, v) & +\nu(D(u), D(v)) \\
& +\lambda(\nabla \cdot(\nabla d \odot \nabla d), v)=0, \\
\left(\partial_{t} d, w\right)+((u \cdot \nabla) d, w) & -\gamma(\Delta d-f(d), w)=0
\end{aligned}\right.
$$

subject to the initial conditions $u(x, 0)=u_{0} \in H$ and $d(x, 0)=d_{0} \in H_{p}^{1}$.

Remark 2.1. For any $v \in V$ and $w, z \in H_{p}^{1}$,

$$
((v \cdot \nabla) z, w)+((v \cdot \nabla) w, z)=0 .
$$

Moreover, by (2.2), for any $v \in H_{p}^{2}$ and $w \in H$,

$$
(\nabla \cdot(\nabla v \odot \nabla v), w)=\left(\Delta v^{T}(\nabla v), w\right) .
$$

Thus the weak form (2.3) is equivalent to the following system:

$$
\left\{\begin{array}{cl}
\left(\partial_{t} u, v\right)+\frac{1}{2}((u \cdot \nabla) u, v) & -\frac{1}{2}((u \cdot \nabla) v, u) \\
& +\nu(D(u), D(v))+\lambda\left(\Delta d^{T}(\nabla d), v\right)=0 \quad \forall v \in V, \\
\left(\partial_{t} d, w\right)+\frac{1}{2}((u \cdot \nabla) d, w) & -\frac{1}{2}((u \cdot \nabla) w, d)+\gamma(\Delta d-f(d), w)=0 \quad \forall w \in H_{p}^{2} .
\end{array}\right.
$$

We now consider a Fourier-spectral method. Let $m^{(j)}=\left(m_{1}^{(j)}, \ldots, m_{n}^{(j)}\right),\left|m^{(j)}\right|=$ $\max _{1 \leq k \leq n}\left|m_{k}^{(j)}\right|$, and $|m|=\max _{1 \leq j \leq n}\left|m^{(j)}\right|$.

Take the standard basis function $\phi_{m}=\left[\phi_{m}^{(1)}, \ldots, \phi_{m}^{(j)}\right]^{T}$ with

$$
\phi_{m}^{(j)}=(2 \pi)^{-\frac{3}{2}} e^{i m^{(j)} \cdot x}, \quad\left|m^{(j)}\right|=0,1,2, \ldots, 1 \leq j \leq n .
$$

Define $\Phi_{M}=\operatorname{span}\left\{\phi_{m}|0 \leq| m \mid \leq M\right\}$. Let $\mathcal{P}_{M}$ be the set consisting of all realvalued functions in $\Phi_{M}$ and $V_{M}=\mathcal{P}_{M} \cap H$. Let $P_{M}: H \rightarrow V_{M}$ be the $L_{p}^{2}$-orthogonal projection. Throughout the paper, we denote by $c$ a generic positive constant independent of any function and $M$.

The Fourier-spectral method for $(2.3)$ is to find a pair $\left(u_{M}, d_{M}\right) \in L^{\infty}\left(0, T ; V_{M}\right) \times$ $L^{\infty}\left(0, T ; \mathcal{P}_{M}\right)$ such that

$$
\left\{\begin{array}{rlrl}
\left(\partial_{t} u_{M}, \phi\right)+\left(\left(u_{M} \cdot \nabla\right) u_{M}, \phi\right)+\nu\left(D\left(u_{M}\right), D(\phi)\right) & & \\
+\lambda\left(\nabla \cdot\left(\nabla d_{M} \odot \nabla d_{M}\right), \phi\right) & =0 & & \forall \phi \in V_{M}, \\
\left(\partial_{t} d_{M}, \psi\right)+\left(\left(u_{M} \cdot \nabla\right) d_{M}, \psi\right)-\gamma\left(\Delta d_{M}-f\left(d_{M}\right), \psi\right) & =0 & & \forall \psi \in \mathcal{P}_{M}
\end{array}\right.
$$

with $u_{M}(x, 0)=P_{M} u_{0}(x)$ and $d_{M}(x, 0)=P_{M} d_{0}(x)$. Note that in general, different values of $M$ may be used for approximating $u$ and $d$.

We first study the existence and uniqueness for the numerical solutions of (2.6). The main result is the following theorem. 
Theorem 2.1. For any $u_{0} \in H, d_{0} \in H_{p}^{1}$ and $T>0$,

1. the problem (2.6) admits a unique weak solution in $Q_{T}$;

2. for any sequence of solutions of (2.6), there exists a subsequence converging to $(u, d)$, a global weak solution of (2.3) in $Q_{T}$.

We shall prove this theorem in the same way as in Lin and Liu [22], with slight modification. Indeed, the periodicity allows us to consider discrete approximations to both $u$ and $d$ directly. This immediately leads to the convergence of numerical solutions; however, a more precise analysis is also required. Furthermore, additional treatment is needed for the untruncated penalty term $f(d)$. The proof is based on the four following propositions.

Proposition 2.2. There exists a $T_{0}>0$ depending only on $u_{0}, d_{0}$, and $M$ such that (2.6) has at least one classical solution in $Q_{T_{0}}$.

Proof. Let $u_{0, m}^{(j)}$ be the $m$ th Fourier coefficient of $u_{0}^{(j)}$ and $c_{1}$ be a suitably large positive constant to be determined below. We choose $y=\left(y^{(1)}, y^{(2)}, \ldots, y^{(n)}\right)^{T}$ in such a way that

$$
\left\{\begin{array}{l}
y^{(j)}(x, t)=\sum_{\left|m_{j}\right|=0}^{M} y_{m}^{(j)}(t) \phi_{m}^{(j)}(x), \\
y_{m}^{(j)}(0)=u_{0, m}^{(j)}, \quad 0 \leq j \leq n,
\end{array}\right.
$$

and $\|y(t)\| \leq c_{1}$ for all $t \in\left[0, T_{1}\right]$. Next, let $z \in \mathcal{P}_{M}$ be the solution of the parabolic equation

$$
\left(\partial_{t} z, \psi\right)+((y \cdot \nabla) z, \psi)-\gamma(\Delta z-f(z), \psi)=0 \quad \forall \psi \in \mathcal{P}_{M}
$$

with $z(x, 0)=P_{M} d_{0}(x)$. Let $g(z)=f(z)+\frac{z}{\epsilon^{2}}$. Clearly, it is monotone. Therefore the theory of the parabolic system (cf. [16]) ensures that (2.7) has a unique solution for a given $y$. By taking $\psi=-\Delta z+P_{M} f(z)$ in (2.7) and integrating by parts, we deduce that for all $t \in\left[0, T_{1}\right]$,

$$
\begin{aligned}
\frac{d}{d t}\left(|z|_{1}^{2}+\right. & \left.2 \int_{\Omega} F(z) d x\right)+2 \gamma\left\|\Delta z-P_{M} f(z)\right\|^{2} \\
& =2\left((y \cdot \nabla) z, \Delta z-P_{M} f(z)\right) \leq \gamma\left\|\Delta z-P_{M} f(z)\right\|^{2}+\frac{1}{\gamma}\|(y \cdot \nabla) z\|^{2} .
\end{aligned}
$$

It is well known (cf. [3] and [14]) that for any $\phi \in \mathcal{P}_{M}$ and $1 \leq p \leq q \leq \infty$,

$$
\|\phi\|_{L^{q}} \leq c M^{\frac{n}{p}-\frac{n}{q}}\|\phi\|_{L^{p}}
$$

and for any $r \geq 0$,

$$
\|\phi\|_{r} \leq c M^{r}\|\phi\| .
$$

Since $y \in V_{M}$, we derive from (2.8) and (2.9) that for all $0 \leq t \leq T_{1}$,

$$
|z(t)|_{1}^{2}+2 \int_{\Omega} F(z) d x \leq e^{\frac{c c_{1}}{\gamma} M^{n} T_{1}}\left(\left|d_{0}\right|_{1}^{2}+2 \int_{\Omega} F\left(d_{0}\right) d x\right) .
$$

Moreover, for any $\phi \in V_{M}$ and all $0 \leq t \leq T_{1}$,

$$
|(\nabla \cdot(\nabla z \odot \nabla z), \phi)| \leq c M^{\frac{n}{2}}|\phi|_{1}|z|_{1}^{2} \leq c_{2}|\phi|_{1},
$$


where $c_{2}$ is a positive constant depending only on $c_{1}, M, \gamma$, and $d_{0}$.

Now let $y^{*}$ be the solution of the system

$$
\left(\partial_{t} y^{*}, \phi\right)+\left(\left(y^{*} \cdot \nabla\right) y^{*}, \phi\right)+\nu\left(D\left(y^{*}\right), D(\phi)\right)+\lambda(\nabla \cdot(\nabla z \odot \nabla z), \phi)=0,
$$

for all $\phi \in V_{M}$ and with $y^{*}(x, 0)=P_{M} u_{0}(x)$. Note that for any $v \in H_{p}^{1}$,

$$
(D(v), D(v)) \geq \frac{1}{2}|v|_{1}^{2}
$$

Hence, (2.12) is a parabolic equation and it has a unique solution. By taking $\phi=y^{*}$ in (2.12) and using (2.4), (2.11), and (2.13), we conclude that for all $t \in\left[0, T_{1}\right]$,

$$
\left\|y^{*}\right\| \leq\left\|P_{M} u_{0}\right\|+c_{3} t \leq\left\|u_{0}\right\|+c_{3} t
$$

with $c_{3}$ being a positive constant depending only on $c_{1}, M, \gamma$, and $d_{0}$.

We now take $c_{1}=2\left(\left\|u_{0}\right\|+1\right)$ and choose a sufficiently small $T_{0}>0$ such that $\left\|u_{o}\right\|+c_{3} T_{0} \leq c_{1}$. Further, we denote the mapping from $y$ to $y^{*}$ by $\mathcal{L}$, that is, $y^{*}=\mathcal{L}(y)$, and set

$$
\mathcal{B}\left(T_{0}\right)=\left\{v \in L^{\infty}\left(0, T_{0} ; V_{M}\right) \mid v(x, 0)=P_{M} u_{0}(x),\|v(t)\| \leq c_{1} \text { for } t \in\left[0, T_{0}\right]\right\} .
$$

As in Lin and Liu [22], it can be shown that $\mathcal{L}$ is a compact mapping from the closed convex set $\mathcal{B}\left(T_{0}\right)$ into $\mathcal{B}\left(T_{0}\right)$. Thus $\mathcal{L}$ has at least one fixed point $u_{M} \in \mathcal{B}\left(T_{0}\right)$. Let $d_{M}$ be the solution governed by the second equation of (2.6). Then $\left(u_{M}, d_{M}\right)$ is a smooth solution of (2.6) in $Q_{T_{0}}$.

It is easy to prove the following technical lemma which is needed in what follows.

Lemma 2.3. Assume that

- $z(t)$ is a nonnegative function of $t$,

- the constants $b_{j} \geq 0, \gamma_{j} \geq 0$,

- $b_{0} e^{N b_{1} t_{1}} \leq \min _{2 \leq j \leq N}\left(\frac{b_{1}}{b_{j}}\right)^{\frac{1}{\gamma_{j}}}$

- $\forall t \in\left[0, t_{1}\right], z(t) \leq \int_{0}^{t} z(s)\left(b_{1}+\sum_{j=2}^{N} b_{j} z^{\gamma_{j}}(s)\right) d s+b_{0}$.

Then for all $t \in\left[0, t_{1}\right]$, we have $z(t) \leq b_{0} e^{N b_{1} t}$.

Proposition 2.4. Let $\left(u_{M}, d_{M}\right)$ be any solution of (2.6). Then for all $t \in\left[0, T_{0}\right]$,

$$
\begin{aligned}
& E(t)+\int_{0}^{t}\left(\frac{\nu}{2}\left|u_{M}(s)\right|_{1}^{2}+2 \lambda \gamma\left\|\Delta d_{M}(s)-P_{M} f\left(d_{M}(s)\right)\right\|^{2}\right) d s \leq \sigma_{0} e^{c_{4} M^{-\frac{1}{8}} t}, \\
& E(t)+\int_{0}^{t}\left(\frac{\nu}{2}\left|u_{M}(s)\right|_{1}^{2}+2 \lambda \gamma\left|d_{M}(s)\right|_{2}^{2}\right) d s \leq\left(1+\frac{4 \gamma}{\epsilon^{2}}\right) \sigma_{0} e^{c_{4} M^{-\frac{1}{8}} t}
\end{aligned}
$$

where

$$
\begin{aligned}
E(t) & =\left\|u_{M}(t)\right\|^{2}+\lambda\left\|d_{M}(t)\right\|_{1}^{2}, \\
\sigma_{0} & =\left\|u_{0}\right\|^{2}+\lambda\left|d_{0}\right|_{1}^{2}+3 \lambda \int_{\Omega} F\left(d_{0}\right) d x+\lambda(2 \pi)^{n}\left(\epsilon^{2}+1+\epsilon \sqrt{\epsilon^{2}+2}\right),
\end{aligned}
$$

and $c_{4}$ is a positive constant depending on $\gamma, \lambda$, and $\epsilon$. 
Proof. We take $\phi=u_{M}$ and $\psi=-\Delta d_{M}+P_{M} f\left(d_{M}\right)$ in (2.6). Using (2.4), (2.5), and (2.13), we obtain that

$$
\frac{d}{d t}\left\|u_{M}\right\|^{2}+\nu\left|u_{M}\right|_{1}^{2}+2 \lambda\left(\left(\Delta d_{M}^{T}\right) \nabla d_{M}, u_{M}\right) \leq 0
$$

and

$$
\begin{aligned}
\frac{d}{d t}\left(\lambda\left|d_{M}\right|_{1}^{2}\right. & \left.+2 \lambda \int_{\Omega} F\left(d_{M}\right) d x\right)-2 \lambda\left(\left(u_{M} \cdot \nabla\right) d_{M}, \Delta d_{M}\right) \\
& +2 \lambda\left(\left(u_{M} \cdot \nabla\right) d_{M}, P_{M} f\left(d_{M}\right)\right)+2 \lambda \gamma\left\|\Delta d_{M}-P_{M} f\left(d_{M}\right)\right\|^{2}=0 .
\end{aligned}
$$

Let $I$ be the identity operator and $g(z)=f(z)+\frac{z}{\epsilon^{2}}$. For any $v \in V$ and $w \in H_{p}^{1}$, we have

$$
((v \cdot \nabla) w, f(w))=0 .
$$

Thus, the combination of (2.14)-(2.16) leads to

$$
\begin{aligned}
\frac{d}{d t}\left(\left\|u_{M}\right\|^{2}+\lambda\left|d_{M}\right|_{1}^{2}\right. & \left.+2 \lambda \int_{\Omega} F\left(d_{M}\right) d x\right)+\nu\left|u_{M}\right|_{1}^{2} \\
& +2 \lambda \gamma\left\|\Delta d_{M}-P_{M} d\left(d_{M}\right)\right\|^{2} \leq 2 \lambda|G|,
\end{aligned}
$$

where

$$
G=\left(\left(u_{M} \cdot \nabla\right) d_{M},\left(P_{M}-I\right) g\left(d_{M}\right)\right) .
$$

For any $v \in H_{p}^{r}$ and $0 \leq \mu \leq r$, we have

$$
\left\|P_{M} v-v\right\|_{\mu} \leq c M^{\mu-r}\|v\|_{r}
$$

Moreover,

$$
\left\|g\left(d_{M}\right)\right\|_{1} \leq c\left\|d_{M}\right\|_{L^{4}}^{2}\left|d_{M}\right|_{1} .
$$

Thus by (2.10) and imbedding theory,

$$
2 \lambda|G| \leq \frac{\nu}{2}\left|u_{M}\right|_{1}^{2}+\frac{c_{4}}{M^{\frac{1}{8}}}\left\|u_{M}\right\|^{2}+\frac{c_{4}}{M^{\frac{3}{8}}}\left\|d_{M}\right\|_{1}^{8} .
$$

Substituting (2.19) into (2.17) and integrating the resulting inequality with respect to $t$, we find that for large $M$,

$$
\begin{aligned}
\left\|u_{M}(t)\right\|^{2}+ & \lambda\left|d_{M}(t)\right|_{1}^{2}+2 \lambda \int_{\Omega} F\left(d_{M}(t)\right) d x \\
& +\int_{0}^{t}\left(\frac{\nu}{2}\left|u_{M}(s)\right|_{1}^{2}+2 \lambda \gamma\left\|\Delta d_{M}(s)-P_{M} f\left(d_{M}(s)\right)\right\|^{2}\right) d s \\
& \leq\left\|u_{0}\right\|^{2}+\lambda\left|d_{0}\right|_{1}^{2}+3 \lambda \int_{\Omega} F\left(d_{0}\right) d x \\
& +\frac{c_{4}}{M^{\frac{1}{8}}} \int_{0}^{t}\left(\left\|u_{M}(s)\right\|^{2}+\frac{1}{M^{\frac{1}{4}}}\left\|d_{M}(s)\right\|_{1}^{8}\right) d s .
\end{aligned}
$$

Clearly,

$$
2 \lambda \int_{\Omega} F\left(d_{M}\right) d x \geq 0 .
$$


Moreover,

$$
\begin{aligned}
2 \lambda \int_{\Omega} F\left(d_{M}\right) d x & \geq \frac{\lambda}{2 \epsilon^{2}}\left(\left\|d_{M}\right\|_{L^{4}}^{4}-2\left\|d_{M}\right\|^{2}+(2 \pi)^{n}\right) \\
& \geq \lambda\left\|d_{M}\right\|^{2}-\frac{\lambda(2 \pi)^{n}}{2}\left(2+\epsilon^{2}\right) .
\end{aligned}
$$

Hence, for some constant $\sigma_{0},(2.20)$ reads

$$
\begin{aligned}
\left\|u_{M}(t)\right\|^{2}+\lambda\left\|d_{M}(t)\right\|_{1}^{2}+ & \int_{0}^{t}\left(\frac{\nu}{2}\left|u_{M}(s)\right|_{1}^{2}+2 \lambda \gamma\left\|\Delta d_{M}(s)-P_{M} f\left(d_{M}(s)\right)\right\|^{2}\right) d s \\
& \leq \sigma_{0}+\frac{c_{4}}{M^{\frac{1}{8}}} \int_{0}^{t}\left(\left\|u_{M}(s)\right\|^{2}+\frac{1}{M^{\frac{1}{4}}}\left\|d_{M}(s)\right\|_{1}^{8}\right) d s .
\end{aligned}
$$

We now use Lemma 2.3 with $N=2, b_{0}=\sigma_{0}, b_{1}=c_{4} M^{-\frac{1}{8}}, b_{2}=c_{4} M^{-\frac{3}{8}}, \gamma_{2}=3$, and $z(t)$ as the left side of (2.22). Then the first conclusion follows immediately.

As in Liu and Walkington [24], a calculation shows that for any $v \in H_{p}^{2}$,

$$
-2 \epsilon^{2}(f(v), \Delta v)=\left\|\nabla\left(|v|^{2}\right)\right\|^{2}+2\||v| \nabla v\|^{2}-2\|\nabla v\|^{2} \geq-2|v|_{1}^{2}
$$

and

$$
\left\|\Delta d_{M}-P_{M} f\left(d_{M}\right)\right\|^{2} \geq\left|d_{M}\right|_{2}^{2}+\left\|P_{M} f\left(d_{M}\right)\right\|^{2}-\frac{2}{\epsilon^{2}}\left|d_{M}\right|_{1}^{2} .
$$

Therefore, by using the previous results, we reach the second conclusion.

Remark 2.2. By Proposition 2.4, for any $\beta>0$, there exist $M_{0}>0$ and $T_{0}>0$ such that for all $M \geq M_{0}$ and all $t \in\left[0, T_{0}\right]$,

$$
E(t) \leq \sigma_{0}(1+\beta t)
$$

Proposition 2.5. Problem (2.6) has a unique weak solution in $Q_{T_{0}}$.

Proof. Suppose that $(2.6)$ has two solutions $\left(u_{M}, d_{M}\right)$ and $\left(\bar{u}_{M}, \bar{d}_{M}\right)$. Let $\tilde{u}_{M}=$ $\bar{u}_{M}-u_{M}$ and $\tilde{d}_{M}=\bar{d}_{M}-d_{M}$. Then

$$
\left\{\begin{array}{rlr}
\left(\partial_{t} \tilde{u}_{M}, \phi\right)+\left(\left(\bar{u}_{M} \cdot \nabla\right) \tilde{u}_{M}, \phi\right)+\nu\left(D\left(\tilde{u}_{M}\right), D(\phi)\right) & \\
+\lambda\left(\nabla \cdot\left(\nabla \tilde{d}_{M} \odot \nabla \tilde{d}_{M}\right), \phi\right)=A(\phi) & & \forall \phi \in V_{M}, \\
\left(\partial_{t} \tilde{d}_{M}, \psi\right)+\left(\left(\tilde{u}_{M} \cdot \nabla\right) \tilde{d}_{M}, \psi\right)-\gamma\left(\Delta \tilde{d}_{M}, \psi\right)=\sum_{j=1}^{2} B_{j}(\psi) & & \forall \psi \in \mathcal{P}_{M},
\end{array}\right.
$$

where

$$
\begin{aligned}
A(\phi) & =-\left(\left(\tilde{u}_{M} \cdot \nabla\right) u_{M}, \phi\right)-2 \lambda\left(\nabla \cdot\left(\nabla d_{M} \odot \nabla \tilde{d}_{M}, \phi\right),\right. \\
B_{1}(\psi) & =-\left(\left(u_{M} \cdot \nabla\right) \tilde{d}_{M}, \psi\right)-\left(\left(\tilde{u}_{M} \cdot \nabla\right) d_{M}, \psi\right), \\
B_{2}(\psi) & =-\gamma\left(f\left(\bar{d}_{M}\right)-f\left(d_{M}\right), \psi\right) .
\end{aligned}
$$

We now denote

$$
\tilde{E}(t)=\left\|\tilde{u}_{M}(t)\right\|^{2}+\left\|\tilde{d}_{M}(t)\right\|^{2}+\lambda\left|\tilde{d}_{M}(t)\right|_{1}^{2}
$$


and take $\phi=2 \tilde{u}_{M}$ and $\psi=-2 \lambda \Delta \tilde{d}_{M}$ in (2.24). In addition, we take $\psi=2 \tilde{d}_{M}$ in the second formula of (2.24). Putting the three results together, we derive from (2.4), (2.5), and (2.13) that

$$
\begin{aligned}
\frac{d \tilde{E}}{d t}+\nu\left|\tilde{u}_{M}\right|_{1}^{2}+2 \gamma\left|\tilde{d}_{M}\right|_{1}^{2}+2 \lambda \gamma\left|\tilde{d}_{M}\right|_{2}^{2} \leq & 2 A\left(\tilde{u}_{M}\right) \\
& +2 \lambda \sum_{j=1}^{2} B_{j}\left(\Delta \tilde{d}_{M}\right)+2 \sum_{j=1}^{2} B_{j}\left(\tilde{d}_{M}\right) .
\end{aligned}
$$

Let $\eta$ be any positive number. Since $\tilde{u}_{M} \in V_{M}$, we have from (2.4) and (2.9) that

$$
\begin{gathered}
\left|A\left(\tilde{u}_{M}\right)\right| \leq \eta\left|\tilde{u}_{M}\right|_{1}^{2}+\frac{c}{\eta} M^{n}\left(\left\|u_{M}\right\|^{2}\left\|\tilde{u}_{M}\right\|^{2}+\left|d_{M}\right|_{1}^{2}\left|\tilde{d}_{M}\right|_{1}^{2}\right), \\
\left|B_{1}\left(\Delta \tilde{d}_{M}\right)\right| \leq \eta\left|\tilde{d}_{M}\right|_{2}^{2}+\frac{c}{\eta} M^{n}\left(\left\|u_{M}\right\|^{2}\left|\tilde{d}_{M}\right|_{1}^{2}+\left|d_{M}\right|_{1}^{2}\left\|\tilde{u}_{M}\right\|^{2}\right) .
\end{gathered}
$$

A direct calculation shows that for any $v, w \in \mathcal{R}^{n}$,

$$
\epsilon^{2}(f(v+w)-f(v)) \cdot w \geq \frac{1}{2}|v|^{2}|w|^{2}-|w|^{2} .
$$

Also,

$$
\begin{aligned}
\epsilon^{2}\left(f\left(\bar{d}_{M}\right)-f\left(d_{M}\right)\right) & =\left(\left|\bar{d}_{M}-d_{M}\right|^{2}-1\right) \tilde{d}_{M}+\left|d_{M}\right|^{2} \tilde{d}_{M} \\
& +\left|\bar{d}_{M}-d_{M}\right| d_{M}\left|\tilde{d}_{M}\right|+2\left(d_{M} \cdot \tilde{d}_{M}\right) \bar{d}_{M} .
\end{aligned}
$$

Thus,

$$
\begin{aligned}
\left|B_{2}\left(\Delta \tilde{d}_{M}\right)\right| & \leq \frac{c}{\epsilon^{2}}\left\|\Delta \tilde{d}_{M}\right\|\left\|\tilde{d}_{M}\right\|_{L^{6}}\left(\left\|\left|\bar{d}_{M}\right|^{2}\right\|_{L^{3}}+\left\|\left|d_{M}\right|^{2}\right\|_{L^{3}}\right)+\frac{1}{\epsilon^{2}}\left\|\Delta \tilde{d}_{M}\right\|\left\|\tilde{d}_{M}\right\| \\
& \leq \eta\left|\tilde{d}_{M}\right|_{2}^{2}+\frac{c}{\eta \epsilon^{4}}\left(\left\|\bar{d}_{M}\right\|_{1}^{4}+\left\|d_{M}\right\|_{1}^{4}+1\right)\left\|\tilde{d}_{M}\right\|_{1}^{2} .
\end{aligned}
$$

From (2.4), (2.9), and (2.26), we have

$$
\left|B_{1}\left(\tilde{d}_{M}\right)\right|+\left|B_{2}\left(\tilde{d}_{M}\right)\right| \leq \frac{\gamma}{\epsilon^{2}}\left\|\tilde{d}_{M}\right\|^{2}+c\left|\tilde{d}_{M}\right|_{1}^{2}+c M^{\frac{n}{3}}\left\|d_{M}\right\|_{1}^{2}\left\|\tilde{u}_{M}\right\|^{2} .
$$

Letting $\eta$ be suitably small, substituting the previous estimates into (2.25), we assert that

$$
\frac{d \tilde{E}}{d t}+\frac{\nu}{2}\left|\tilde{u}_{M}\right|_{1}^{2}+\gamma\left|\tilde{d}_{M}\right|_{1}^{2}+\lambda \gamma\left|\tilde{d}_{M}\right|_{2}^{2} \leq c_{5}\left(\left\|\tilde{u}_{M}\right\|^{2}+\left\|\tilde{d}_{M}\right\|_{1}^{2}\right),
$$

where $c_{5}$ is a positive constant depending only on $\nu, \lambda, \gamma, \epsilon, M,\left\|u_{M}\right\|,\left\|d_{M}\right\|_{1}$, and $\left\|\bar{d}_{M}\right\|_{1}$. This fact, together with $\tilde{u}_{M}(x, 0)=\tilde{d}_{M}(x, 0)=0$, leads to the conclusion.

Proposition 2.6. For any $T>0$, problem (2.6) has a unique weak solution in $Q_{T}$.

Proof. Let $c_{6}(t)=\sigma_{0}(1+\beta t)$. If $\left(u_{M}, d_{M}\right)$ is a solution of $(2.6)$ in $Q_{T_{0}}$, then by Remark 2.3,

$$
\left\|u_{M}\left(T_{0}\right)\right\|^{2}+\left\|d_{M}\left(T_{0}\right)\right\|_{1}^{2} \leq c_{6}\left(T_{0}\right) .
$$


Take $c_{1}=c_{6}\left(T_{0}\right)$ in the proof of Proposition 2.2. Then there exists a positive constant $\delta$ depending only on $c_{6}\left(T_{0}\right)$ and $M$ such that (2.6) has a unique solution $\left(\bar{u}_{M}, \bar{d}_{M}\right)$ in $\mathcal{R}^{n} \times\left[T_{0}, T_{0}+\delta\right]$ with $\bar{u}_{M}\left(T_{0}\right)=u_{M}\left(T_{0}\right)$ and $\bar{u}_{M}\left(T_{0}\right)=d_{M}\left(T_{0}\right)$. Finally, the conclusion follows from the extension property.

To study the convergence of (2.6), as well as the existence and the uniqueness of global weak solution of (2.3), we recall the following lemma [23].

Lemma 2.7. Assume that

- $X_{0} \subset X \subset X_{1}$ are three Hilbert spaces,

- the injections $X_{0} \rightarrow X$ and $X \rightarrow X_{1}$ are continuous,

- the injection $X_{0} \rightarrow X$ is compact,

- $Y=L^{\alpha_{0}}\left(0, T ; X_{0}\right) \cap W^{1, \alpha_{1}}\left(0, T ; X_{1}\right), \alpha_{0}, \alpha_{1}>1$.

Then the injection $Y \rightarrow L^{\alpha_{0}}(0, T ; X)$ is also compact.

The next lemma can be proved by adapting the same line of argument as for Theorem 3.2 in Temam [30] to the periodic setting.

Lemma 2.8. If $v_{k} \rightarrow v$ weakly in $L^{2}(0, T ; V)$ and strongly in $L^{2}(0, T ; H)$, then

$$
\int_{Q_{T}}\left(v_{k} \cdot \nabla\right) v_{k} \cdot w d x \rightarrow \int_{Q_{T}}(v \cdot \nabla) v \cdot w d x \quad \forall w \in L^{2}(0, T ; H) .
$$

We are now in position to prove Theorem 2.1.

Proof of Theorem 2.1. Let $\left\{\left(u_{M}, d_{M}\right)\right\}$ be a sequence of solutions of (2.6) in $Q_{T}$. For any $v \in H$, we take $\phi=P_{M} v$ in the first formula of (2.6). Since $n \leq 3$, we use (2.4) and imbedding theory to obtain that

$$
\left|\left(\partial_{t} u_{M}, \phi\right)\right| \leq c\|\phi\|_{1}\left(\left\|u_{M}\right\|_{1}+\left\|u_{M}\right\|^{\frac{1}{2}}\left\|u_{M}\right\|_{1}^{\frac{3}{2}}+\left\|d_{M}\right\|_{1}^{\frac{1}{2}}\left\|d_{M}\right\|_{2}^{\frac{3}{2}}\right),
$$

which with Proposition 2.4 implies that

$$
\left\|\partial_{t} u_{M}\right\|_{L^{\frac{4}{3}}\left(0, T ; H^{-1}\right)} \leq c_{7}(T)
$$

with $c_{7}(T)$ being independent of $M$. Thus by Lemma 2.7, $\left\{u_{M}\right\}$ is precompact in $L^{2}(0, T ; H)$. Similarly, $\left\{d_{M}\right\}$ is precompact in $L^{2}\left(0, T ; H_{p}^{1}\right)$.

We now take a subsequence, still denoted by $\left\{\left(u_{M}, d_{M}\right)\right\}$, such that

- $u_{M} \rightarrow u$, weakly in $L^{2}(0, T ; V)$, weak we $_{*}^{\infty}(0, T ; H)$, and strongly in $L^{2}(0, T ; H)$

- $d_{M} \rightarrow d$, weakly in $L^{2}\left(0, T ; H_{p}^{2}\right)$, weak we $_{*} L^{\infty}\left(0, T ; H_{p}^{1}\right)$, and strongly in $L^{2}\left(0, T ; H_{p}^{1}\right)$.

A standard process shows that $(u, d)$ is a weak solution of (2.3). Finally, the previous statements indicate that for all $t \in[0, T]$,

$$
\|u(t)\|^{2}+\|d(t)\|_{1}^{2}+\int_{0}^{t}\left(|u(s)|_{1}^{2}+|d(s)|_{2}^{2}\right) d s \leq \frac{2}{\min (4 \lambda \gamma, \nu)}\left(1+\frac{4 \gamma}{\epsilon^{2}}\right) \sigma_{0} e^{c_{4} M^{-\frac{1}{8}} t} .
$$

The proof of Theorem 2.1 is then complete.

TheOrem 2.9. For $n=2$, the whole sequence $\left\{\left(u_{M}, d_{M}\right)\right\}$ converges to $(u, d)$, the unique solution of (2.3) in $Q_{T}$. 
Proof. It suffices to prove the uniqueness of the global weak solution of (2.3). Suppose that both $(\bar{u}, \bar{d})$ and $(u, d)$ are solutions of $(2.3)$. Let $\tilde{u}=\bar{u}-u$ and $\tilde{d}=\bar{d}-d$. We can derive an energy estimate for $(\tilde{u}, \tilde{d})$, which is exactly of the same form as $(2.25)$. The only difference is that $u_{M}, d_{M}, \tilde{u}_{M}$, and $\tilde{d}_{M}$ in (2.25) are now replaced by $u, d, \tilde{u}$ and $\tilde{d}$, respectively. The terms $A(\tilde{u}), B_{j}(\Delta \tilde{d})$, and $B_{j}(\tilde{d})$ can be estimated as before. Finally we get

$$
\frac{d}{d t}\left(\|\tilde{u}\|^{2}+\|\tilde{d}\|_{1}^{2}\right) \leq c\left(\|u\|_{1}^{2}+\|\bar{d}\|_{2}^{2}+\|d\|_{2}^{2}+\|\bar{d}\|_{1}^{4}+\|d\|_{1}^{4}\right)\left(\|\tilde{u}\|^{2}+\|\tilde{d}\|_{1}^{2}\right) .
$$

Let

$$
\sigma(t)=-c \int_{0}^{t}\left(\|u\|_{1}^{2}+\|\bar{d}\|_{2}^{2}+\|d\|_{2}^{2}+\|\bar{d}\|_{1}^{4}+\|d\|_{1}^{4}\right) d s .
$$

By virtue of Theorem 2.1, $\sigma(t)$ is finite for all $t \in[0, T]$. Then the conclusion follows from this fact with $\tilde{u}(x, 0)=\tilde{d}(x, 0)=0$.

Remark 2.3. Theorem 2.1 also provides a proof for the existence of the global weak solution of (2.3). Theorem 2.9 gives the uniqueness of solution for $n=2$. The uniqueness of solution of (2.3) with $n=3$ is an open problem just as in the NavierStokes equations. Whereas by an argument similar to Theorem 3.4 of Temam [30], we can prove that (2.3) has at most one solution such that

$$
\begin{aligned}
& u \in L^{2}(0, T ; V) \cap L^{8}\left(0, T ; L_{p}^{4}\right) \cap L^{\infty}(0, T ; H), \\
& d \in L^{2}\left(0, T ; H_{p}^{2}\right) \cap L^{8}\left(0, T ; W_{p}^{1,4}\right) \cap L^{\infty}\left(0, T ; H_{p}^{1}\right) .
\end{aligned}
$$

In general, we can follow the same line as in Lions [23] to show the uniqueness of solution $(u, d)$ of $(2.3)$ if

$$
\begin{aligned}
& u \in L^{2}(0, T ; V) \cap L^{s}\left(0, T ; L_{p}^{r}\right) \cap L^{\infty}(0, T ; H), \\
& d \in L^{2}\left(0, T ; H_{p}^{2}\right) \cap L^{s}\left(0, T ; W_{p}^{1, r}\right) \cap L^{\infty}\left(0, T ; H_{p}^{1}\right)
\end{aligned}
$$

where $\frac{2}{s}+\frac{n}{r} \leq 1$. Such a solution is continuous from $[0, T]$ into $H \times H_{p}^{1}$.

Remark 2.4. The energy estimate in Theorem 2.1 depends on $\epsilon$. However, if $F\left(d_{0}\right) \leq c$, then for all $t \in[0, T]$, we have

$$
\left\|u_{M}(t)\right\|^{2}+\left\|d_{M}(t)\right\|_{1}^{2}+\nu \int_{0}^{t}\left|u_{M}(s)\right|_{1}^{2} d s \leq c_{8}(T),
$$

where $c_{8}(T)$ is independent of $\epsilon$.

Remark 2.5. When $\Omega$ is a bounded domain with a piecewise smooth boundary and the Dirichlet boundary condition is imposed, we can consider a modified Fourier-Galerkin method as in Lin and Liu [22], and deal with the terms involving (untruncated) $f(d)$ as in this section, to derive the same results as in Theorems 2.1 and 2.9 .

Remark 2.6. By combining the techniques used in this section and those in Lin and Liu [22], we can derive the existence, the uniqueness, and the regularities of the local classical solution of (2.3) for $n=2,3$. 
Remark 2.7. In [21], the liquid crystal model is generalized to non-Newtonian flows by replacing the first equation in (1.1) with

$$
\frac{\partial u}{\partial t}+(u \cdot \nabla) u-\nu \nabla \cdot D^{*}(u)+\nabla p+\lambda \nabla \cdot(\nabla d \odot \nabla d)=0, \quad x \in \Omega, 0<t \leq T,
$$

where $D^{*}(u)=q(|D(u)|) D(u), q(z)=c_{0}+c_{1} z^{r}$, for some $c_{0}, c_{1}$ and $r \geq 1 / 2$, and $D(u)=\left(\nabla u+(\nabla u)^{T}\right) / 2$. In this case, we may follow the same line of arguments as above and as in [17] to derive the regularity results as in Remark 2.3 for the solutions $(u, d)$. Then, as in Remark 2.3, we can also show that the solution $(u, d)$ is unique.

3. Error estimates. In the last section, we proved that the numerical solutions tend to weak solutions of (2.3) in a suitable sense. We shall prove in this section that the scheme (2.6) provides the spectral accuracy for the unique smooth solution. The main difficulties in the error estimate come from the following three facts: (i) the system (2.6) is a strongly nonlinear system, requiring delicate, nontrivial nonlinear analysis as in [12]; (ii) some conservation properties of the numerical solution play important roles for the convergence of weak solutions; however, the disturbed system for the error functions does not satisfy the same conservation properties; (iii) the system (2.6) is not of parabolic type. Thus, the error estimates cannot be easily derived from a straightforward application of usual approaches. Therefore, a detailed error analysis as presented below is fully justified.

THEOREM 3.1. We assume that

$$
\begin{aligned}
u \in L^{2}\left(0, T ; H_{p}^{r_{1}}\right) \cap L^{\infty}\left(0, T ; H \cap H_{p}^{\sigma_{1}}\right), & \sigma_{1}>\frac{n}{2}, \\
d \in L^{2}\left(0, T ; H_{p}^{r_{2}}\right) \cap L^{\infty}\left(0, T ; H_{p}^{\sigma_{2}}\right), & \sigma_{2}>\frac{n}{2}+1,
\end{aligned}
$$

with $r_{1}>0$ and $r_{2}>1$. Then, for all $t \in[0, T]$,

$$
\begin{aligned}
& \left\|u-u_{M}\right\|_{L^{2}\left(0, T ; H_{p}^{1}\right) \cap L^{\infty}\left(0, T ; L_{p}^{2}\right)} \leq c^{*}\left(M^{-r_{1}}+M^{1-r_{2}}\right), \\
& \left\|d-d_{M}\right\|_{L^{2}\left(0, T ; H_{p}^{2}\right) \cap L^{\infty}\left(0, T ; H_{p}^{1}\right)} \leq c^{*}\left(M^{-r_{1}}+M^{1-r_{2}}\right),
\end{aligned}
$$

where $c^{*}$ is a positive constant depending only on $\nu, \lambda, \gamma, \epsilon$, and the norms of $u$ and $d$ in the spaces mentioned in (3.1).

Proof. Let $u_{M}^{*}=P_{M} u, d_{M}^{*}=P_{M} d, \tilde{u}_{M}=u_{M}-u_{M}^{*}$, and $\tilde{d}_{M}=d_{M}-d_{M}^{*}$. Then it follows from (2.3) and (2.6) that for all $t \in[0, T]$ and $(\phi, \psi) \in V_{M} \times \mathcal{P}_{M}$,

$$
\left\{\begin{aligned}
\left(\partial_{t} \tilde{u}_{M}, \phi\right)+\left(\left(u_{M} \cdot \nabla\right) \tilde{u}_{M}, \phi\right) & +\nu\left(D\left(\tilde{u}_{M}\right), D(\phi)\right) \\
& +\lambda\left(\nabla \cdot\left(\nabla \tilde{d}_{M} \odot \nabla \tilde{d}_{M}\right), \phi\right)=\sum_{j=1}^{4} A_{j}(\phi), \\
\left(\partial_{t} \tilde{d}_{M}, \psi\right)+\left(\left(\tilde{u}_{M} \cdot \nabla\right) \tilde{d}_{M}, \psi\right) & -\gamma\left(\Delta \tilde{d}_{M}-f\left(\tilde{d}_{M}\right), \psi\right)=\sum_{j=1}^{8} B_{j}(\psi),
\end{aligned}\right.
$$


where

$$
\begin{aligned}
& A_{1}(\phi)=\left((u \cdot \nabla) u-\left(u_{M}^{*} \cdot \nabla\right) u_{M}^{*}, \phi\right), \\
& A_{2}(\phi)=\lambda\left(\nabla \cdot(\nabla d \odot \nabla d, \phi)-\left(\nabla \cdot\left(\nabla d_{M}^{*} \odot \nabla d_{M}^{*}\right), \psi\right),\right. \\
& A_{3}(\phi)=-\left(\left(\tilde{u}_{M} \cdot \nabla\right) u_{M}^{*}, \phi\right), \\
& A_{4}(\phi)=-2 \lambda\left(\nabla \cdot\left(\nabla \tilde{d}_{M} \odot \nabla d_{M}^{*}\right), \phi\right), \\
& B_{1}(\psi)=-\left((u \cdot \nabla) d-\left(u_{M}^{*} \cdot \nabla\right) d_{M}^{*}, \psi\right), \\
& B_{2}(\psi)=-\gamma\left(f(d)-f\left(d_{M}^{*}\right), \psi\right), \\
& B_{3}(\psi)=-\left(\left(\tilde{u}_{M} \cdot \nabla\right) d_{M}^{*}, \psi\right), \\
& B_{4}(\psi)=-\left(\left(u_{M}^{*} \cdot \nabla\right) \tilde{d}_{M}, \psi\right), \\
& B_{5}(\psi)=-\frac{\gamma}{\epsilon^{2}}\left(\left|d_{M}^{*}\right|^{2} \tilde{d}_{M}, \psi\right), \\
& B_{6}(\psi)=-\frac{2 \gamma}{\epsilon^{2}}\left(\left(d_{M}^{*} \cdot \tilde{d}_{M}\right) d_{M}^{*}, \psi\right), \\
& B_{7}(\psi)=-\frac{\gamma}{\epsilon^{2}}\left(\left|\tilde{d}_{M}\right|^{2} d_{M}^{*}, \psi\right), \\
& B_{8}(\psi)=-\frac{2 \gamma}{\epsilon^{2}}\left(\left(d_{M}^{*} \cdot \tilde{d}_{M}\right) \tilde{d}_{M}, \psi\right) .
\end{aligned}
$$

We first take $\phi=\tilde{u}_{M}$ in (3.2). Using (2.4), (2.5), and (2.13), we obtain that

$$
\frac{d}{d t}\left\|\tilde{u}_{M}\right\|^{2}+\nu\left|\tilde{u}_{M}\right|_{1}^{2}+2 \lambda\left(\Delta \tilde{d}_{M}^{T}\left(\nabla \tilde{d}_{M}\right), \tilde{u}_{M}\right) \leq 2 \sum_{j=1}^{4}\left|A_{j}\left(\tilde{u}_{M}\right)\right| .
$$

Let $g(v)=f(v)+v / \epsilon^{2}$ and $\tilde{G}=\left(\left(\tilde{u}_{M} \cdot \nabla\right) \tilde{d}_{M},\left(P_{M}-I\right) g\left(\tilde{d}_{M}\right)\right)$. By taking $\psi=\tilde{\psi}_{M}=-\Delta \tilde{d}_{M}+P_{M} f\left(\tilde{d}_{M}\right)$ in (3.2), we derive from (2.4) and (2.16) that

$$
\begin{aligned}
\frac{d}{d t}\left(\lambda\left|\tilde{d}_{M}\right|_{1}^{2}\right. & \left.+2 \lambda \int_{\Omega} F\left(\tilde{d}_{M}\right) d x\right)-2 \lambda\left(\left(\tilde{u}_{M} \cdot \nabla\right) \tilde{d}_{M}, \Delta \tilde{d}_{M}\right)+2 \lambda \gamma\left\|\tilde{\psi}_{M}\right\|^{2} \\
& \leq 2 \lambda \sum_{j=1}^{8}\left|B_{j}\left(\tilde{\psi}_{M}\right)\right|+2 \lambda|\tilde{G}| .
\end{aligned}
$$

To derive an energy estimate, we have to take only $\psi=\tilde{\psi}$ in (3.2). However, in order to derive an error estimate, a more precise estimate for $\left\|\tilde{d}_{M}\right\|$ is required. To this end, we rewrite the second formula of (3.2) in the following alternative form:

$$
\left(\partial_{t} \tilde{d}_{M}, \psi\right)+\left(\left(u_{M} \cdot \nabla\right) \tilde{d}_{M}, \psi\right)-\gamma\left(\Delta \tilde{d}_{M}, \psi\right)+\gamma\left(f\left(d_{M}\right)-f\left(d_{M}^{*}\right), \psi\right)=\sum_{j=1}^{3} B_{j}(\psi) .
$$

By taking $\psi=\tilde{d}_{M}$ in (3.5) and using (2.4) and (2.26), we get

$$
\frac{2 \lambda}{\epsilon^{2}} \frac{d}{d t}\left\|\tilde{u}_{M}\right\|^{2}+\frac{4 \lambda \gamma}{\epsilon^{2}}\left|\tilde{d}_{M}\right|_{1}^{2} \leq \frac{4 \lambda \gamma}{\epsilon^{4}}\left\|\tilde{d}_{M}\right\|^{2}+\frac{4 \lambda}{\epsilon^{2}} \sum_{j=1}^{3}\left|B_{j}\left(\tilde{d}_{M}\right)\right| .
$$


Let

$$
E^{*}(t)=\left\|\tilde{u}_{M}(t)\right\|^{2}+\frac{2 \lambda}{\epsilon^{2}}\left\|\tilde{d}_{M}(t)\right\|^{2}+\lambda\left|\tilde{d}_{M}(t)\right|_{1}^{2}+2 \lambda \int_{\Omega} F\left(\tilde{d}_{M}(t)\right) d x .
$$

The combination of (3.3), (3.4), and (3.6) leads to

$$
\begin{aligned}
\frac{d E^{*}}{d t}+\nu\left|\tilde{u}_{M}\right|_{1}^{2}+\frac{4 \lambda \gamma}{\epsilon^{2}}\left|\tilde{d}_{M}\right|_{1}^{2}+2 \lambda \gamma\left\|\tilde{\psi}_{M}\right\|^{2} & \leq \frac{4 \lambda \gamma}{\epsilon^{4}}\left\|\tilde{d}_{M}\right\|^{2}+2 \sum_{j=1}^{4}\left|A_{j}\left(\tilde{u}_{M}\right)\right| \\
+\sum_{j=1}^{8}\left|B_{j}\left(\tilde{\psi}_{M}\right)\right| & +\frac{4 \lambda}{\epsilon^{2}} \sum_{j=1}^{3}\left|B_{j}\left(\tilde{d}_{M}\right)\right|+2 \lambda|\tilde{G}| .
\end{aligned}
$$

It remains to estimate the terms at the right side of (3.7). We first estimate $\left|A_{j}\left(\tilde{u}_{M}\right)\right|$. Due to (2.4) and (2.18),

$$
\begin{gathered}
\left|A_{1}\left(\tilde{u}_{M}\right)\right| \leq \eta\left|\tilde{u}_{M}\right|_{1}^{2}+\frac{c}{\eta} M^{-2 r_{1}}\|u\|_{\sigma_{1}}^{2}\|u\|_{r_{1}}^{2}, \\
\left|A_{2}\left(\tilde{u}_{M}\right)\right| \leq \eta\left|\tilde{u}_{M}\right|_{1}^{2}+\frac{c}{\eta} M^{2-2 r_{2}}\|d\|_{\sigma_{2}}^{2}\|d\|_{r_{2}}^{2}, \\
\left|A_{3}\left(\tilde{u}_{M}\right)\right| \leq \eta\left|\tilde{u}_{M}\right|_{1}^{2}+\frac{c}{\eta}\left(\|u\|^{2}\|u\|_{1}^{6}+\|u\|^{\frac{1}{2}}\|u\|_{1}^{\frac{3}{2}}\right)\left\|\tilde{u}_{M}\right\|^{2}, \\
\left|A_{4}\left(\tilde{u}_{M}\right)\right| \leq \eta\left(\left|\tilde{u}_{M}\right|_{1}^{2}+\left|\tilde{d}_{M}\right|_{2}^{2}\right)+c\left(\frac{1}{\eta^{7}}\|d\|_{1}^{2}\|d\|_{2}^{6}+\frac{1}{\eta}\|d\|_{1}^{\frac{1}{2}}\|d\|_{2}^{\frac{3}{2}}\right)\left\|\tilde{d}_{M}\right\|_{1}^{2} .
\end{gathered}
$$

Next, we estimate $\left|B_{j}\left(\tilde{\psi}_{M}\right)\right|$. Using (2.18) and imbedding theory yields

$$
\left|B_{1}\left(\tilde{\psi}_{M}\right)\right| \leq \eta \|\left.\tilde{\psi}_{M}\right|^{2}+\frac{c}{\eta}\left(M^{-2 r_{1}}+M^{2-2 r_{2}}\right)\left(\|u\|_{\sigma_{2}}^{2}\|d\|_{r_{2}}^{2}+\|u\|_{r_{1}}^{2}\|d\|_{\sigma_{2}}^{2}\right) .
$$

Similar to (2.27),

$$
\begin{aligned}
\epsilon^{2}\left(f\left(d_{M}^{*}\right)-f(d)\right)= & \left(\left|d_{M}^{*}-d\right|^{2}-1\right)\left(d_{M}^{*}-d\right)+|d|^{2}\left(d_{M}^{*}-d\right) \\
& +\left|d_{M}^{*}-d\right|^{2} d+2\left(d_{M}^{*} \cdot\left(d_{M}^{*}-d\right)\right) d_{M}^{*} .
\end{aligned}
$$

Thus, by (2.18) and imbedding theory,

$$
\left|B_{2}\left(\tilde{\psi}_{M}\right)\right| \leq \eta\left\|\tilde{\psi}_{M}\right\|^{2}+\frac{c}{\eta \epsilon^{4}} M^{-2 r_{2}}\left(\|d\|_{\sigma_{1}}^{4}+1\right)\|d\|_{r_{2}}^{2} .
$$

It is easy to show that

$$
\begin{gathered}
\left|B_{3}\left(\tilde{\psi}_{M}\right)\right| \leq \eta\left(\left\|\tilde{\psi}_{M}\right\|^{2}+\left|\tilde{u}_{M}\right|_{1}^{2}\right)+c\left(\frac{1}{\eta^{7}}\|d\|_{1}^{2}\|d\|_{2}^{6}+\frac{1}{\eta}\|d\|_{1}^{\frac{1}{2}}\|d\|_{2}^{\frac{3}{2}}\right)\left\|\tilde{u}_{M}\right\|^{2}, \\
\left|B_{4}\left(\tilde{\psi}_{M}\right)\right| \leq \eta\left(\left\|\tilde{\psi}_{M}\right\|^{2}+\left|\tilde{d}_{M}\right|_{2}^{2}\right)+c\left(\frac{1}{\eta^{7}}\|u\|^{2}\|u\|_{1}^{6}+\frac{1}{\eta}\|u\|^{\frac{1}{2}}\|u\|_{1}^{\frac{3}{2}}\right)\left\|\tilde{d}_{M}\right\|_{1}^{2},
\end{gathered}
$$




$$
\left|B_{j}\left(\tilde{\psi}_{M}\right)\right| \leq \eta\left\|\tilde{\psi}_{M}\right\|^{2}+\frac{c}{\eta \epsilon^{4}}\|d\|_{\sigma_{1}}^{4}\left\|\tilde{d}_{M}\right\|^{2}, \quad j=5,6,7,8 .
$$

We now estimate $\left|B_{j}\left(\tilde{d}_{M}\right)\right|$. We use (2.5), (2.18), and (2.26) to show that

$$
\begin{gathered}
\left|B_{1}\left(\tilde{d_{M}}\right)\right| \leq c\left|\tilde{d}_{M}\right|_{1}^{2}+c\left(M^{-2 r_{1}}+M^{-2 r_{2}}\right)\left(\|u\|_{\sigma_{1}}^{2}\|d\|_{r_{2}}^{2}+\|u\|_{r_{1}}^{2}\|d\|_{\sigma_{1}}^{2}\right), \\
\left|B_{2}\left(\tilde{d}_{M}\right)\right| \leq c\left\|\tilde{d}_{M}\right\|^{2}+\frac{c}{\epsilon^{4}} M^{-2 r_{2}}\|d\|_{\sigma_{1}}^{4}\|d\|_{r_{2}}^{2}, \\
\left|B_{3}\left(\tilde{d}_{M}\right)\right| \leq c\left|\tilde{d}_{M}\right|_{1}^{2}+c\|d\|_{\sigma_{1}}^{2}\left\|\tilde{u}_{M}\right\|^{2} .
\end{gathered}
$$

Finally, we estimate $|\tilde{G}|$. Similar to (2.19), we have

$$
|\tilde{G}| \leq \eta\left(\left|\tilde{u}_{M}\right|_{1}^{2}+\left|\tilde{d}_{M}\right|_{2}^{2}\right)+\frac{c}{\epsilon^{2} M}\left(\left\|\tilde{u}_{M}\right\|^{2}+\left\|\tilde{d}_{M}\right\|_{1}^{8}\right)+\frac{c}{\eta^{6} \epsilon^{8} M^{7}}\left\|\tilde{d}_{M}\right\|_{1}^{26} .
$$

Besides,

$$
\left\|\tilde{\psi}_{M}\right\|^{2} \geq \frac{1}{2}\left|\tilde{d}_{M}\right|_{2}^{2}-\frac{c}{\epsilon^{4}}\left\|\tilde{d}_{M}\right\|_{L^{6}}^{6} \geq \frac{1}{2}\left|\tilde{d}_{M}\right|_{2}^{2}-\frac{1}{\epsilon^{4}}\left\|\tilde{d}_{M}\right\|_{1}^{6}-\frac{c}{\epsilon^{4}}\left\|\tilde{d}_{M}\right\|_{1}^{2} .
$$

Letting $\eta$ be suitably small, taking $\sigma_{1}<2$, and substituting (3.8)-(3.21) into (3.7), we obtain that

$$
\frac{d E^{*}}{d t}+\frac{\nu}{2}\left|\tilde{u}_{M}\right|_{1}^{2}+\lambda \gamma\left|\tilde{d}_{M}\right|_{2}^{2} \leq F_{1}\left(\tilde{u}_{M}, \tilde{d}_{M}\right)+F_{2}\left(\tilde{d}_{M}\right)+F_{3}(u, d),
$$

where

$$
\begin{aligned}
F_{1}\left(\tilde{u}, \tilde{d}_{M}\right)= & b_{1}^{*}\left(\left\|\tilde{u}_{M}\right\|^{2}+\left\|\tilde{d}_{M}\right\|_{1}^{2}+\left\|\tilde{d}_{M}\right\|_{1}^{4}\right), \\
F_{2}\left(\tilde{d}_{M}\right)= & c_{\epsilon} M^{-1}\left\|\tilde{d}_{M}\right\|_{1}^{8}+c_{2} M^{-7}\left\|\tilde{d}_{M}\right\|_{1}^{26}, \\
F_{3}(u, d)= & c_{\epsilon}\left(M^{-2 r_{1}}+M^{2-2 r_{2}}\right)\left(\|u\|_{\sigma_{1}}^{2}\|u\|_{r_{1}}^{2}+\|d\|_{\sigma_{2}}^{2}\|d\|_{r_{2}}^{2}+\|u\|_{r_{1}}^{2}\|d\|_{\sigma_{2}}^{2}\right. \\
& \left.+\|u\|_{\sigma_{1}}^{2}\|d\|_{r_{2}}^{2}+\|d\|_{\sigma_{1}}^{4}\|d\|_{r_{2}}^{2}+\|d\|_{r_{2}}^{2}\right),
\end{aligned}
$$

and $c_{\epsilon}$ is a positive constant depending only on $\mu, \lambda, \gamma$, and $\epsilon$, and $b_{1}^{*}$ is a positive constant depending only on $\|u\|_{1},\|d\|_{2}$ and $c_{\epsilon}$. We derive by direct calculation that

$$
F\left(\tilde{d}_{M}\right)=\frac{1}{4 \epsilon^{2}}\left|\tilde{d}_{M}\right|^{4}-\frac{1}{2 \epsilon^{2}}\left|\tilde{d}_{M}\right|^{2}+\frac{1}{4 \epsilon^{2}}(2 \pi)^{n} .
$$

Let us denote

$$
\tilde{E}(t)=\left\|\tilde{u}_{M}(t)\right\|^{2}+\frac{\lambda}{\epsilon^{2} 2}\left\|\tilde{d}_{M}(t)\right\|^{2}+\lambda\left|\tilde{d}_{M}(t)\right|_{1}^{2}+\frac{\lambda}{2 \epsilon^{2}}\left\|\tilde{d}_{M}(t)\right\|_{L^{4}}^{4} .
$$

Clearly,

$$
E^{*}(t) \geq \tilde{E}(t)+\frac{\lambda}{2 \epsilon^{2}}(2 \pi)^{n}, \quad E^{*}(0)=\frac{\lambda}{2 \epsilon^{2}}(2 \pi)^{n} .
$$

Thus, integrating (3.22) with respect to $t$ and using (3.23) and (3.24) yield that

$$
\begin{aligned}
\tilde{E}(t)+\frac{\nu}{2} \int_{0}^{t}\left|\tilde{u}_{M}(s)\right|_{1}^{2} d s+\lambda \gamma \int_{0}^{t}\left|\tilde{d}_{M}(s)\right|_{2}^{2} d s \leq & \int_{0}^{t}\left(F_{1}\left(\tilde{u}_{M}(s), \tilde{d}_{M}(s)\right)\right. \\
& \left.+F_{2}\left(\tilde{d}_{M}(s)\right)\right) d s+\rho(t),
\end{aligned}
$$


where

$$
\rho(t)=\int_{0}^{t} F_{3}(u(s), d(s)) d s \leq b_{2}^{*}\left(M^{-2 r_{1}}+M^{2-2 r_{2}}\right)
$$

and $b_{2}^{*}$ is a positive constant depending only on $\nu, \lambda, \gamma, \epsilon, T$ and the norms $\|u\|_{L^{2}\left(0, T ; H_{p}^{r_{1}}\right)}$, $\|u\|_{L^{\infty}\left(0, T ; H_{p}^{\sigma_{1}}\right)},\|d\|_{L^{2}\left(0, T ; H_{p}^{\left.r_{2}\right)}\right.}$, and $\|d\|_{L^{\infty}\left(0, T ; H_{p}^{\sigma_{2}}\right)}$.

We now apply Lemma 2.3 to the last inequality with $N=4, b_{0}=\rho(T), b_{1}=$ $O(1), b_{2}=O(1), b_{3}=O\left(M^{-1}\right), b_{4}=O\left(M^{-7}\right), \gamma_{2}=1, \gamma_{3}=3, \gamma_{4}=12$, and

$$
z(t)=\tilde{E}(t)+\frac{\nu}{2} \int_{0}^{t}\left|\tilde{u}_{M}(s)\right|_{1}^{2} d s+\lambda \gamma \int_{0}^{t}\left|\tilde{d}_{M}(s)\right|_{2}^{2} d s .
$$

Since $r_{1}>0$ and $r_{2}>1$, we have $\rho \rightarrow 0$ as $M \rightarrow \infty$. Thus, all conditions of Lemma 2.3 are fulfilled and the desired result follows.

Remark 3.1. Theorem 3.1 indicates the spectral accuracy of the Fourier-spectral approximation (2.6) for a smooth solution. It also shows that if a global weak solution of (2.3) possesses the regularity as in (3.1), then it is a unique solution.

4. Fully discrete Fourier spectral approximations. In actual computation, we have to discretize the derivatives of unknown functions with respect to $t$ in (2.6). To do this, let $\tau$ be the mesh size of $t$ and

$$
\Pi_{T}=\left\{t=k \tau, 0 \leq k \leq\left[\frac{T}{\tau}\right]\right\} .
$$

For any $t \in \Pi_{T}$, we denote the value of $v(x, t+\tau)$ by $v^{+}(x, t)$ or $v^{+}$for simplicity. Let

$$
\bar{v}=\frac{1}{2}\left(v^{+}+v\right), \quad \delta_{t} v=\frac{1}{\tau}\left(v^{+}-v\right) .
$$

Clearly,

$$
2 \delta_{t} v \cdot v=\delta_{t}|v|^{2}-\tau\left|\delta_{t} v\right|^{2}, \quad 2 \delta_{t} v \cdot \bar{v}=\delta_{t}|v|^{2} .
$$

Let $0 \leq \theta \leq 1$. A fully discrete scheme for (2.3) is to find a pair $\left(u_{M}, d_{M}\right)$ for all $t \in \Pi_{T}$ such that for all $(\phi, \psi) \in V_{M} \times \mathcal{P}_{M}$,

$$
\begin{cases}\left(\delta_{t} u_{M}, \phi\right)+\left(\left(u_{M} \cdot \nabla\right) u_{M}, \phi\right)+\nu\left(D \left(u_{M}\right.\right. & \left.\left.+\theta \tau \delta_{t} u_{M}\right), D(\phi)\right) \\ & +\lambda\left(\nabla \cdot\left(\nabla d_{M} \odot \nabla d_{M}\right), \phi\right)=0 \\ \left(\delta_{t} d_{M}, \psi\right)+\left(\left(u_{M} \cdot \nabla\right) d_{M}, \psi\right)-\gamma\left(\Delta \left(d_{M}\right.\right. & \left.\left.+\theta \tau \delta_{t} d_{M}\right)-f\left(d_{M}\right), \psi\right)=0\end{cases}
$$

subject to $u_{M}(x, 0)=P_{M} u_{0}(x)$ and $d_{M}(x, 0)=P_{M} d_{0}(x)$.

Remark 4.1. In (4.2), the linear terms are approximated by the usual $\theta$ scheme which is explicit if $\theta=0$, otherwise it is implicit. For the sake of efficiency, the nonlinear terms in (4.2) are treated explicitly, so the stability condition (4.15) is required.

The following lemma, which is a special case of Lemma 4.16 of Guo [13], will be used to estimate the time discretization error.

Lemma 4.1. Suppose that

- $z(t)$ and $y(z)$ are nonnegative functions defined on $\Pi_{T}$, and $y(z) \leq 0$ for $z \leq \alpha$ 
- the constants $\alpha>0$ and $b_{j} \geq 0$,

- $b_{0} e^{N b_{1} T} \leq \min \left(\alpha, \min _{2 \leq j \leq N}\left(\frac{b_{1}}{b_{j}}\right)^{\frac{1}{\gamma_{j}}}\right)$, and

- $\forall t \in \Pi_{T}, z(t) \leq \tau \sum_{\substack{s \in \Pi_{T} \\ s<t}}\left(z(s)\left(b_{1}+\sum_{j=2}^{N} b_{j} z^{\gamma_{j}}(s)\right)+y(s)\right)+b_{0}$.

Then for all $t \in \Pi_{T}$, we have $z(t) \leq b_{0} e^{N b_{1} t}$.

Let $u_{M}^{*}=P_{M} u, d_{M}^{*}=P_{M} d, \tilde{u}_{M}=u_{M}-u_{M}^{*}$, and $\tilde{d}_{M}=d_{M}-d_{M}^{*}$. Like (3.2), we have the following error equations:

$$
\left\{\begin{aligned}
\left(\delta_{t} \tilde{u}_{M}, \phi\right) & +\left(\left(u_{M} \cdot \nabla\right) \tilde{u}_{M}, \phi\right)+\nu\left(D\left(\tilde{u}_{M}+\theta \tau \delta_{t} \tilde{u}_{M}\right), D(\phi)\right) \\
& +\lambda\left(\nabla \cdot\left(\nabla \tilde{d}_{M} \odot \nabla \tilde{d}_{M}\right), \phi\right)=\sum_{j=0}^{4} A_{j}(\phi) \quad \forall \phi \in V_{M}, \\
\left(\delta_{t} \tilde{d}_{M}, \psi\right) & +\left(\left(\tilde{u}_{M} \cdot \nabla\right) \tilde{d}_{M}, \psi\right) \\
& -\gamma\left(\Delta\left(\tilde{d}_{M}+\theta \tau \delta_{t} \tilde{d}_{M}\right)-f\left(\tilde{d}_{M}\right), \psi\right)=\sum_{j=0}^{8} B_{j}(\psi) \quad \forall \psi \in \mathcal{P}_{M},
\end{aligned}\right.
$$

where for $j \geq 1, A_{j}(\phi)$ and $B_{j}(\psi)$ are the same as in (3.2), and

$$
A_{0}(\phi)=\left(\partial_{t} u-\delta_{t} u, \phi\right), \quad B_{0}(\psi)=\left(\partial_{t} d-\delta_{t} d, \psi\right) .
$$

We first take $\phi=\tilde{u}_{M}$ in (4.3). Then it follows from (2.4), (2.5), (2.13), and (4.1) that

$$
\begin{aligned}
\delta_{t}\left\|\tilde{u}_{M}\right\|^{2}-\tau\left\|\delta_{t} \tilde{u}_{M}\right\|^{2}+\nu\left|\tilde{u}_{M}\right|_{1}^{2} & +2 \theta \nu \tau\left(D\left(\delta_{t} \tilde{u}_{M}\right), D\left(\tilde{u}_{M}\right)\right) \\
& +2 \lambda\left(\Delta \tilde{d}_{M}\left(\nabla \tilde{d}_{M}\right), \tilde{u}_{M}\right) \leq 2 \sum_{j=0}^{4}\left|A_{j}\left(\tilde{u}_{M}\right)\right| .
\end{aligned}
$$

Next, let $\xi$ be a positive number to be determined. We take $\phi=\xi \tau \delta_{t} \tilde{u}_{M}$ in (4.3) and then by (2.13),

$$
\xi \tau\left\|\delta_{t} \tilde{u}_{M}\right\|^{2}+\frac{1}{2} \xi \theta \nu \tau^{2}\left|\delta_{t} \tilde{u}_{M}\right|_{1}^{2}+\xi \nu \tau\left(D\left(\tilde{u}_{M}\right), D\left(\delta_{t} \tilde{u}_{M}\right)\right) \leq \xi \tau \sum_{j=0}^{7}\left|A_{j}\left(\delta_{t} \tilde{u}_{M}\right)\right|,
$$

where

$$
\begin{aligned}
& A_{5}\left(\delta_{t} \tilde{u}_{M}\right)=-\left(\left(u_{M}^{*} \cdot \nabla\right) \tilde{u}_{M}, \delta_{t} \tilde{u}_{M}\right), \\
& A_{6}\left(\delta_{t} \tilde{u}_{M}\right)=-\left(\left(\tilde{u}_{M} \cdot \nabla\right) \tilde{u}_{M}, \delta_{t} \tilde{u}_{M}\right), \\
& A_{7}\left(\delta_{t} \tilde{u}_{M}\right)=-\lambda\left(\nabla \cdot\left(\nabla \tilde{d}_{M} \odot \nabla \tilde{d}_{M}\right), \delta_{t} \tilde{u}_{M}\right) .
\end{aligned}
$$

Now we take $\psi=-\lambda \Delta \tilde{d}_{M}$ in (4.3). By (2.4), (2.23), and (4.1), we obtain that

$$
\begin{aligned}
\lambda \delta_{t}\left|\tilde{d}_{M}\right|_{1}^{2} & -\lambda \tau\left|\delta_{t} \tilde{d}_{M}\right|_{1}^{2}-2 \lambda\left(\left(\tilde{u}_{M} \cdot \nabla\right) \tilde{d}_{M}, \Delta \tilde{d}_{M}\right)+2 \lambda \gamma\left|\tilde{d}_{M}\right|_{2}^{2}+\theta \lambda \gamma \delta_{t}\left|\tilde{d}_{M}\right|_{2}^{2} \\
& -\theta \lambda \gamma \tau^{2}\left|\delta_{t} \tilde{d}_{M}\right|_{2}^{2} \leq \frac{2 \lambda \gamma}{\epsilon^{2}}\left|\tilde{d}_{M}\right|_{1}^{2}+2 \lambda \sum_{j=0}^{8}\left|B_{j}\left(\Delta \tilde{u}_{M}\right)\right| .
\end{aligned}
$$

Moreover, taking $\psi=-\xi \lambda \tau \Delta \delta_{t} \tilde{d}_{M}$ in (4.3) and using (4.1), we deduce that

$$
\begin{aligned}
\xi \lambda \tau\left|\delta_{t} \tilde{d}_{M}\right|_{1}^{2} & +\frac{1}{2} \xi \lambda \gamma \tau \delta_{t}\left|\tilde{d}_{M}\right|_{2}^{2}-\frac{1}{2} \xi \lambda \gamma \tau^{2}\left|\delta_{t} \tilde{d}_{M}\right|_{2}^{2} \\
& +\xi \theta \lambda \gamma \tau^{2}\left|\delta_{t} \tilde{d}_{M}\right|_{2}^{2} \leq \xi \lambda \tau \sum_{j=0}^{10}\left|B_{j}\left(\Delta \delta_{t} \tilde{d}_{M}\right)\right|,
\end{aligned}
$$


where

$$
\begin{aligned}
B_{9}\left(\Delta \delta_{t} \tilde{d}_{M}\right) & =-\left(\left(\tilde{u}_{M} \cdot \nabla\right) \tilde{d}_{M}, \Delta \delta_{t} \tilde{d}_{M}\right), \\
B_{10}\left(\Delta \delta_{t} \tilde{d}_{M}\right) & =-\gamma\left(f\left(\tilde{d}_{M}\right), \Delta \delta_{t} \tilde{d}_{M}\right) .
\end{aligned}
$$

We now rewrite the second formula of (4.3) as follows:

$$
\begin{aligned}
\left(\delta_{t} \tilde{d}_{M}, \psi\right)+\left(\left(u_{M} \cdot \nabla\right) \tilde{d}_{M}, \psi\right) & -\gamma\left(\Delta\left(\tilde{d}_{M}+\theta \tau \delta_{t} \tilde{u}_{M}\right), \psi\right) \\
& +\gamma\left(f(d)-d\left(d_{M}^{*}\right), \psi\right)=\sum_{j=0}^{3} B_{j}(\psi) .
\end{aligned}
$$

Taking $\psi=\tilde{d}_{M}$ in (4.8) and using (2.4), (2.26), and (4.1), we assert that

$$
\begin{aligned}
\delta_{t}\left\|\tilde{d}_{M}\right\|^{2}- & \tau\left\|\delta_{t} \tilde{d}_{M}\right\|^{2}+2 \gamma\left|\tilde{d}_{M}\right|_{1}^{2}+\theta \gamma \tau \delta_{t}\left|\tilde{d}_{M}\right|_{1}^{2}-\theta \gamma \tau^{2}\left|\delta_{t} \tilde{d}_{M}\right|_{1}^{2} \\
& \leq \frac{2 \gamma}{\epsilon^{2}}\left\|\tilde{d}_{M}\right\|^{2}+2 \sum_{j=0}^{3}\left|B_{j}\left(\tilde{d}_{M}\right)\right| .
\end{aligned}
$$

Finally, taking $\psi=\xi \tau \delta_{t} \tilde{d}_{M}$ in (4.3), we derive from (4.1) that

$$
\begin{aligned}
\xi \tau\left\|\delta_{t} \tilde{d}_{M}\right\|^{2}+\frac{1}{2} \xi \gamma \tau \delta_{t}\left|\tilde{d}_{M}\right|_{1}^{2} & -\frac{1}{2} \xi \gamma \tau^{2}\left|\delta_{t} \tilde{d}_{M}\right|_{1}^{2}+\xi \theta \gamma \tau^{2}\left|\delta_{t} \tilde{d}_{M}\right|_{1}^{2} \\
& =\xi \tau \sum_{j=0}^{10}\left|B_{j}\left(\delta_{t} \tilde{d}_{M}\right)\right| .
\end{aligned}
$$

It can be verified by the definition of $D(v)$ and (4.1) that

$$
\begin{aligned}
\nu \tau\left(D\left(\tilde{u}_{M}\right), D\left(\delta_{t} \tilde{u}_{M}\right)\right) & \geq \frac{1}{4} \nu \tau \delta_{t}\left(\left|\tilde{u}_{M}\right|_{1}^{2}+\left\|\partial_{x_{1}} \tilde{u}_{M}^{(1)}\right\|^{2}+\left\|\partial_{x_{2}} \tilde{u}_{M}^{(2)}\right\|^{2}\right) \\
& -\frac{1}{2} \nu \tau^{2}\left|\delta_{t} \tilde{u}_{M}\right|_{1}^{2}-\frac{1}{4} \nu\left|\tilde{u}_{M}\right|_{1}^{2} .
\end{aligned}
$$

Let

$$
\begin{aligned}
E^{*}(t)= & \left\|\tilde{d}_{M}(t)\right\|^{2}+\frac{1}{4} \nu \tau(2 \theta+\xi)\left(\left|\tilde{u}_{M}(t)\right|_{1}^{2}+\left\|\partial_{x_{1}} \tilde{u}_{M}^{(1)}(t)\right\|^{2}+\left\|\partial_{x_{2}} \tilde{u}_{M}^{(2)}(t)\right\|^{2}\right) \\
& +\left(\lambda+\theta \gamma \tau+\frac{1}{2} \xi \gamma \tau\right)\left|\tilde{d}_{M}(t)\right|_{1}^{2}+\lambda \gamma \tau\left(\theta+\frac{\xi}{2}\right)\left|\tilde{d}_{M}(t)\right|_{2}^{2}+\left\|\tilde{u}_{M}\right\|^{2}
\end{aligned}
$$

Putting (4.4)-(4.7) and (4.9)-(4.10) together and using (4.11), we derive that

$$
\delta_{t} E^{*}+2 \gamma\left|\tilde{d}_{M}\right|_{1}^{2}+2 \lambda \gamma\left|\tilde{d}_{M}\right|_{2}^{2}+\sum_{j=1}^{4} \alpha_{j} \leq F_{0},
$$

where

$$
\begin{aligned}
& \alpha_{1}=\nu\left(1-\frac{1}{2} \theta-\frac{1}{4} \xi\right)\left|\tilde{u}_{M}\right|_{1}^{2}, \\
& \alpha_{2}=\tau(\xi-1)\left\|\delta_{t} \tilde{u}_{M}\right\|^{2}+\frac{1}{2} \nu \tau^{2}(\xi \theta-2 \theta-\xi)\left|\delta_{t} \tilde{u}_{M}\right|_{1}^{2}, \\
& \alpha_{3}=\tau(\xi-1)\left\|\delta_{t} \tilde{d}_{M}\right\|^{2}+\gamma \tau^{2}\left(\xi \theta-\theta-\frac{1}{2} \xi\right)\left|\delta_{t} \tilde{d}_{M}\right|_{1}^{2}, \\
& \alpha_{4}=\lambda \tau(\xi-1)\left|\delta_{t} \tilde{d}_{M}\right|_{1}^{2}+\lambda \gamma \tau^{2}\left(\xi \theta-\theta-\frac{1}{2} \xi\right)\left|\delta_{t} \tilde{d}_{M}\right|_{2}^{2},
\end{aligned}
$$


and

$$
\begin{aligned}
F_{0}= & \frac{2 \gamma}{\epsilon^{2}}\left\|\tilde{d}_{M}\right\|^{2}+\frac{2 \lambda \gamma}{\epsilon^{2}}\left|\tilde{d}_{M}\right|_{1}^{2}+2 \sum_{j=0}^{4}\left|A_{j}\left(\tilde{u}_{M}\right)\right|+\xi \tau \sum_{j=0}^{7}\left|A_{j}\left(\delta_{t} \tilde{u}_{M}\right)\right| \\
& +2 \lambda \sum_{j=0}^{8}\left|B_{j}\left(\Delta \tilde{d}_{M}\right)\right|+\xi \lambda \tau \sum_{j=0}^{10}\left|B_{j}\left(\Delta \delta_{t} \tilde{d}_{M}\right)\right| \\
& +2 \sum_{j=0}^{3}\left|B_{j}\left(\tilde{d}_{M}\right)\right|+\xi \tau \sum_{j=0}^{10}\left|B_{j}\left(\delta_{t} \tilde{d}_{M}\right)\right| .
\end{aligned}
$$

We now choose the value of $\xi$ such that all $\alpha_{j}$ are positive. Indeed, for any $\phi \in \mathcal{P}_{M}$,

$$
|\phi|_{1}^{2} \leq n M^{2}\|\phi\|^{2} .
$$

Let $\delta$ be a suitably small positive number and

$$
\xi_{1}(\theta, \delta)=\frac{1+\theta \nu n \tau M^{2}+\delta}{1+\frac{\nu}{2} n \tau M^{2}(\theta-1)}, \quad \xi_{2}(\theta, \delta)=\frac{1+\theta \gamma n \tau M^{2}+\delta}{1+\gamma n \tau M^{2}\left(\theta-\frac{1}{2}\right)} .
$$

Suppose that for $\theta<\frac{1}{2}$, we have

$$
\tau<\tau_{1}(\theta)=\frac{1}{n M^{2}} \min \left(\frac{2}{\gamma(1-\theta)}, \frac{3-2 \theta}{\nu\left((1-\theta)^{2}+1\right)}, \frac{2}{\gamma(1-2 \theta)}, \frac{3-2 \theta}{2 \gamma(\theta-1)^{2}}\right),
$$

and for $\frac{1}{2} \leq \theta \leq 1$, we have

$$
\tau<\tau_{2}(\theta)=\frac{1}{n M^{2}} \min \left(\frac{1}{\nu(1-\theta)}, \frac{3-2 \theta}{\nu\left((1-\theta)^{2}+1\right)}, \frac{3-2 \theta}{2 \gamma(\theta-1)^{2}}\right) .
$$

Thanks to (4.15), we can take $\xi=\max \left(\xi_{1}(\theta, \delta), \xi_{2}(\theta, \delta)\right) \leq 4-2 \theta-4 \delta$. Therefore,

$$
\alpha_{1} \geq \delta \nu\left|\tilde{u}_{M}\right|_{1}^{2} .
$$

By (4.13) and (4.14),

$$
\begin{gathered}
\alpha_{2} \geq \tau(\xi-1)\left\|\delta_{t} \tilde{u}_{M}\right\|^{2}+\frac{1}{2} \nu n \tau^{2} M^{2}(\xi \theta-2 \theta-\xi)\left\|\delta_{t} \tilde{u}_{M}\right\|^{2} \geq \delta \tau\left\|\delta_{t} \tilde{u}_{M}\right\|^{2}, \\
\alpha_{3} \geq \delta \tau\left\|\delta_{t} \tilde{d}_{M}\right\|^{2}, \quad \alpha_{4} \geq \lambda \delta \tau\left|\delta_{t} \tilde{d}_{M}\right|_{1}^{2} .
\end{gathered}
$$

Therefore, (4.12) reads

$$
\begin{aligned}
\delta_{t} E^{*}+\delta \nu\left|\tilde{u}_{M}\right|_{1}^{2} & +2 \gamma\left|\tilde{d}_{M}\right|_{1}^{2}+2 \lambda \gamma\left|\tilde{d}_{M}\right|_{2}^{2} \\
& +\delta \tau\left(\left\|\delta_{t} \tilde{u}_{M}\right\|^{2}+\left\|\delta_{t} \tilde{d}_{M}\right\|^{2}+\lambda\left|\delta_{t} \tilde{d}_{M}\right|_{1}^{2}\right) \leq F_{0} .
\end{aligned}
$$

Hence, it suffices to estimate the terms in $F_{0}$.

Let $\eta, \sigma_{1}, \sigma_{2}, r_{1}$, and $r_{2}$ have the same meanings as in the last section. We first estimate $\left|A_{j}\left(\tilde{u}_{M}\right)\right|$. Clearly,

$$
\left|A_{0}\left(\tilde{u}_{M}\right)\right| \leq c\left\|\tilde{u}_{M}\right\|^{2}+c \tau^{2}\|u\|_{H^{2}\left(t, t+\tau, L_{p}^{2}\right)}^{2} .
$$


For $1 \leq j \leq 4$, the terms $\left|A_{j}\left(\tilde{u}_{M}\right)\right|$ have been estimated in (3.8)-(3.11). We now estimate $\xi \tau\left|A_{j}\left(\delta_{t} \tilde{u}_{M}\right)\right|$. Obviously,

$$
\xi \tau\left|A_{0}\left(\delta_{t} \tilde{u}_{M}\right)\right| \leq \eta \tau\left\|\delta_{t} \tilde{u}_{M}\right\|^{2}+\frac{c}{\eta} \tau^{3}\|u\|_{H^{2}\left(t, t+\tau ; L_{p}^{2}\right)}^{2} .
$$

Since $\delta_{t} \tilde{u}_{M} \in V_{M}$, we use (2.4), (2.9), (2.10), (2.18), (4.15), and imbedding theory to get

$$
\begin{aligned}
\xi \tau\left|A_{1}\left(\delta_{t} \tilde{u}_{M}\right)\right|+\xi \tau\left|A_{2}\left(\delta_{t} \tilde{u}_{M}\right)\right| \leq & \eta \tau\left\|\delta_{t} \tilde{u}_{M}\right\|^{2}+\frac{c}{\eta} M^{-2 r_{1}}\|u\|_{\sigma_{1}}^{2}\|u\|_{r_{1}}^{2} \\
& +\frac{c}{\eta} M^{2-2 r_{2}}\|d\|_{\sigma_{2}}^{2}\|d\|_{r_{2}}^{2}, \\
\xi \tau\left|A_{3}\left(\delta_{t} \tilde{u}_{M}\right)\right|+\xi \tau\left|A_{5}\left(\delta_{t} \tilde{u}_{M}\right)\right| \leq & \eta \tau\left\|\delta_{t} \tilde{u}_{M}\right\|^{2}+\eta\left|\tilde{u}_{M}\right|_{1}^{2} \\
& +c\left(\frac{1}{\eta^{7}}\|u\|^{2}\|u\|_{1}^{6}+\frac{1}{\eta}\|u\|^{\frac{1}{2}}\|u\|_{1}^{\frac{3}{2}}\right)\left\|\tilde{u}_{M}\right\|^{2}, \\
\xi \tau\left|A_{4}\left(\delta_{t} \tilde{u}_{M}\right)\right| \leq & \eta \tau\left\|\delta_{t} \tilde{u}_{M}\right\|^{2}+\eta\left|\tilde{d}_{M}\right|_{2}^{2} \\
& +c\left(\frac{1}{\eta^{7}}\|d\|_{1}^{2}\|d\|_{2}^{6}+\frac{1}{\eta}\|d\|_{1}^{\frac{1}{2}}\|d\|_{2}^{\frac{3}{2}}\right)\left\|\tilde{d}_{M}\right\|_{1}^{2} . \\
\xi \tau\left|A_{6}\left(\delta_{t} \tilde{u}_{M}\right)\right|+\xi \tau\left|A_{7}\left(\delta_{t} \tilde{u}_{M}\right)\right| \leq \eta \tau & \delta_{t} \tilde{u}_{M} \|^{2}+\frac{c}{\eta} M^{n-2}\left(\left\|\tilde{u}_{M}\right\|^{2}\left|\tilde{u}_{M}\right|_{1}^{2}+\left\|\tilde{d}_{M}\right\|_{1}^{2}\left|\tilde{d}_{M}\right|_{2}^{2}\right) .
\end{aligned}
$$

Next, we estimate $\left|B_{j}\left(\Delta \tilde{d}_{M}\right)\right|$. Clearly,

$$
\left|B_{0}\left(\Delta \tilde{d}_{M}\right)\right| \leq \eta\left|\tilde{d}_{M}\right|_{2}^{2}+\frac{c \tau^{2}}{\eta}\|d\|_{H^{2}\left(t, t+\tau ; L_{p}^{2}\right)}^{2} .
$$

For $1 \leq j \leq 8,\left|B_{j}\left(\Delta \tilde{d}_{M}\right)\right|$ can be estimated as in (3.12)-(3.16). The only difference is that $\tilde{\psi}_{M}$ in those estimations are now replaced by $\Delta \tilde{d}_{M}$. Therefore,

$$
\sum_{j=1}^{8}\left|B_{j}\left(\Delta \tilde{d}_{M}\right)\right| \leq \eta\left|\tilde{u}_{M}\right|_{1}^{2}+\eta\left|\tilde{d}_{M}\right|_{2}^{2}+G\left(\tilde{u}_{M}, \tilde{d}_{M}\right),
$$

where

$$
\begin{aligned}
G\left(\tilde{u}_{M}, \tilde{d}_{M}\right)= & c_{\epsilon}\left(\frac{1}{\eta^{7}}\|d\|_{1}^{\frac{1}{2}}\|d\|_{2}^{\frac{3}{2}}+\frac{1}{\eta}\|d\|_{1}^{2}\|d\|_{2}^{6}\right)\left\|\tilde{u}_{M}\right\|^{2} \\
& +c_{\epsilon}\left(\frac{1}{\eta^{7}}\|u\|^{\frac{1}{2}}\|u\|_{1}^{\frac{3}{2}}+\frac{1}{\eta}\|u\|_{1}^{2}\|u\|_{1}^{6}\right)\left\|\tilde{d}_{M}\right\|_{1}^{2} \\
& +\frac{c_{\epsilon}}{\eta^{2}}\left(\|d\|_{\sigma_{1}}^{4}\left\|\tilde{d}_{M}\right\|^{2}+\|d\|_{\sigma_{1}}^{2}\left\|\tilde{d}_{M}\right\|_{1}^{4}\right) \\
& +\frac{c_{\epsilon}}{\eta}\left(M^{-2 r_{1}}+M^{2-2 r_{2}}\right)\left(\|u\|_{\sigma_{1}}^{2}\|d\|_{r_{2}}^{2}+\|u\|_{r_{1}}^{2}\|d\|_{\sigma_{2}}^{2}\right) \\
& +\frac{c_{\epsilon}}{\eta} M^{-2 r_{2}}\left(\|d\|_{\sigma_{1}}^{4}+1\right)\|d\|_{r_{2}}^{2}
\end{aligned}
$$

We now estimate $\xi \tau\left|B_{j}\left(\Delta \delta_{t} \tilde{d}_{M}\right)\right|$. By (2.9), (2.10), and (4.15), we have that

$$
\xi \tau\left|B_{0}\left(\Delta \delta_{t} \tilde{d}_{M}\right)\right| \leq \eta \tau\left|\delta_{t} \tilde{d}_{M}\right|_{1}^{2}+\frac{c \tau^{2}}{\eta}\|d\|_{H^{2}\left(t, t+\tau ; L_{p}^{2}\right)}^{2}
$$




$$
\begin{aligned}
& \xi \tau \sum_{j=1}^{8}\left|B_{j}\left(\Delta \delta_{t} \tilde{d}_{M}\right)\right| \leq \eta\left|\tilde{u}_{M}\right|_{1}^{2}+\eta \tau\left|\delta_{t} \tilde{d}_{M}\right|_{1}^{2}+G\left(\tilde{u}_{M}, \tilde{d}_{M}\right) \\
& \xi \tau\left|B_{9}\left(\Delta \delta_{t} \tilde{d}_{M}\right)\right|+\xi \tau\left|B_{10}\left(\Delta \delta_{t} \tilde{d}_{M}\right)\right| \leq \eta \tau\left|\delta_{t} \tilde{d}_{M}\right|_{1}^{2}+\frac{c}{\eta} M^{n}\left\|\tilde{u}_{M}\right\|^{2}\left|\tilde{d}_{M}\right|_{1}^{2} \\
& +\frac{c_{\epsilon}}{\eta}\left(\left\|\tilde{d}_{M}\right\|_{1}^{6}+\left\|\tilde{d}_{M}\right\|^{2}\right) .
\end{aligned}
$$

The terms $\left|B_{j}\left(\tilde{d}_{M}\right)\right|$ have been estimated by (3.17)-(3.19). Finally, we can estimate $\xi \tau\left|B_{j}\left(\delta_{t} d_{M}\right)\right|$ in the same manner as before to obtain that

$$
\begin{aligned}
\xi \tau \sum_{j=0}^{10}\left|B_{j}\left(\delta_{t} \tilde{d}_{M}\right)\right| \leq & \left\|\tilde{u}_{M}\right\|^{2}+\eta\left|\tilde{u}_{M}\right|_{1}^{2}+\eta \tau\left\|\delta_{t} \tilde{d}_{M}\right\|^{2}+\frac{c}{\eta} M^{n-2}\left\|\tilde{u}_{M}\right\|^{2}\left\|\tilde{d}_{M}\right\|_{1}^{2} \\
& +\frac{c_{\epsilon}}{\eta} M^{-2}\left\|\tilde{d}_{M}\right\|_{1}^{6}+\tau G\left(\tilde{u}_{M}, \tilde{d}_{M}\right) .
\end{aligned}
$$

Letting $\eta$ be suitably small and inserting the previous estimates into (4.19), we derive that for some $\delta_{1}>0$,

$\delta_{t} E^{*}+\left(\delta_{1}-c M^{n-2}\left\|\tilde{u}_{M}\right\|^{2}\right)\left|\tilde{u}_{M}\right|_{1}^{2}+\left(\delta_{1}-M^{n-2}\left\|\tilde{d}_{M}\right\|_{1}^{2}\right)\left|\tilde{d}_{M}\right|_{2}^{2} \leq F_{4}\left(\tilde{u}_{M}, \tilde{d}_{M}\right)+F_{5}(u, d)$,

where

$$
\begin{aligned}
F_{4}\left(\tilde{u}_{M}, \tilde{d}_{M}\right) & =b_{1}^{*}\left(\left\|\tilde{u}_{M}\right\|^{2}+\left\|\tilde{d}_{M}\right\|_{1}^{2}+\left\|\tilde{d}_{M}\right\|_{1}^{4}\right)+\left\|\tilde{d}_{M}\right\|_{1}^{6}+c_{\epsilon} M^{n}\left\|\tilde{u}_{M}\right\|^{2}\left\|\tilde{d}_{M}\right\|_{1}^{2}, \\
F_{5}(u, d) & =F_{3}(u, d)+c \tau^{2}\left(\|u\|_{H^{2}\left(t, t+\tau ; L_{p}^{2}\right)}^{2}+\|d\|_{H^{2}\left(t, t+\tau ; L_{p}^{2}\right)}^{2}\right),
\end{aligned}
$$

and $b_{1}^{*}$ and $F_{3}(u, d)$ being the same as in (3.22).

Now, let $\tilde{E}(t)=\left\|\tilde{u}_{M}\right\|^{2}+\left\|\tilde{u}_{M}\right\|_{1}^{2}$. By summing (4.20) for $t \in \Pi_{T}$, we derive that

$$
\begin{gathered}
\tilde{E}(t)+\tau \sum_{\substack{s \in \Pi_{T} \\
s<t}}\left[\left(\delta_{1}-M^{n-2}\left\|\tilde{u}_{M}(s)\right\|^{2}\right)+\left(\delta_{1}-c M^{n-2}\left\|\tilde{d}_{M}(s)\right\|_{1}^{2}\right)\left|\tilde{d}_{M}(s)\right|_{2}^{2}\right] \\
\quad \leq c \tau \sum_{\substack{s \in \Pi_{T} \\
s<t}} F_{4}\left(\tilde{u}_{M}(s), \tilde{d}_{M}(s)\right)+\rho(t)
\end{gathered}
$$

where

$$
\rho(t)=c \tau \sum_{\substack{s \in \Pi_{T} \\ s<t}} F_{5}(u(s), d(s))=b^{*}\left(\tau^{2}+M^{-2 r_{1}}+M^{2-2 r_{2}}\right),
$$

and $b^{*}$ is a positive constant depending on $\nu, \lambda, \gamma, \epsilon$, and the norms $\|u\|_{C\left(0, T ; H_{p}^{r_{1}}\right)}$, $\|d\|_{C\left(0, T ; H_{p}^{r_{2}}\right)},\|u\|_{H^{2}\left(0, T ; L_{p}^{2}\right)}$, and $\|d\|_{H^{2}\left(0, T ; L_{p}^{2}\right)}$, provided that

$$
r_{1}>\frac{n}{2}, \quad r_{2}>\frac{n}{2}+1
$$


We can now apply Lemma 4.1 to $(4.21)$ with $z(t)=\tilde{E}(t)$ and

$$
y(t)=\left(c M^{n-2}\left\|\tilde{u}_{M}(t)\right\|^{2}-\delta_{1}\right)\left|\tilde{u}_{M}(t)\right|_{1}^{2}+\left(c M^{n-2}\left\|\tilde{d}_{M}(t)\right\|_{1}^{2}-\delta_{1}\right)\left|\tilde{d}_{M}(t)\right|_{2}^{2} .
$$

The corresponding parameters are $N=4, \alpha=O\left(M^{n-2}\right), b_{0}=\rho(t)=O\left(\tau^{2}+M^{-2 r_{1}}+\right.$ $\left.M^{2-2 r_{2}}\right), b_{1}=O(1), b_{2}=O(1), b_{3}=O(1), b_{4}=O\left(M^{n}\right), \gamma_{2}=1, \gamma_{3}=2$, and $\gamma_{4}=1$. In particular, we have $\rho(t)=o\left(M^{n}\right)$ thanks to (4.22). Therefore, we have proved the following result.

THEOREM 4.2. If (4.15) and (4.22) hold, and $u \in C\left(0, T ; H_{p}^{r_{1}}\right) \cap H^{2}\left(0, T ; L_{p}^{2}\right)$ and $d \in C\left(0, T ; H_{p}^{r_{2}}\right) \cap H^{2}\left(0, T ; L_{p}^{2}\right)$, then, for all $t \in \Pi_{T}$,

$$
\begin{aligned}
\left\|u(t)-u_{M}(t)\right\|^{2}+\left\|d(t)-d_{M}(t)\right\|_{1}^{2} & +\tau \sum_{\substack{s \in \Pi_{T} \\
s<t}}\left(\left|u(s)-u_{M}(s)\right|_{1}^{2}+\left|d(s)-d_{M}(s)\right|_{2}^{2}\right) \\
& \leq c^{*}\left(\tau^{2}+M^{-2 r_{1}}+M^{2-2 r_{2}}\right),
\end{aligned}
$$

where $c^{*}$ is a positive constant depending on $\nu, \lambda, \gamma, \epsilon$, and the norms of $u$ and $d$ in the mentioned spaces.

5. Numerical results. In this section, we present some numerical simulations of the model equation (1.1) using the numerical schemes presented in section 4. As our main purpose is to demonstrate the effectiveness of the numerical methods we have studied, the simulations are restricted to very special cases.

In all the simulations, we have chosen the geometry to be $[-\pi, \pi]$ with parameters $\nu=\gamma=1$ and $\lambda=10$. The parameter $\epsilon$ in the nonlinear terms $f$ is set to be 0.1 . We take zero to be the initial velocity.

In our numerical simulations, the number of Fourier modes in each variable is taken to be 32 first with a time step size of $10^{-3}$, and then compared to the results computed with 64 modes and a time step size of $0.5 \times 10^{-3}$. In all the testing cases, the convergence of the numerical solution is evident and we present only the numerical results with 32 modes in order to better present the graphics (vector plots with large number of nodes are difficult to visualize when being reduced in size for presentation).

5.1. +1-degree defect at the origin. We begin with the case of a +1-degree for the director field at the origin, i.e., the initial director field is given by

$$
d_{0}(x, y)=0.2 * \sin ((x+\pi) / 2)^{2} \sin ((y+\pi) / 2)^{2}(x-y, x+y) .
$$

The plots of the director field and the velocity field are given in Figure 5.1 and Figure 5.2, respectively. In addition, the norm of the velocity field is plotted as a function of time. It shows that the presence of the defect generates a transition velocity field. After the annihilation of the defect that came from the boundary, the velocity field turns to zero eventually and the director field approaches to a constant state. The initial velocity generation process is not greatly affected by the periodic boundary conditions, but the latter annihilation process is caused by the periodicity assumption.

In fact, in all the test runs we report here, the solutions always converge to some steady state which consists of a trivial velocity field and a constant director field. It remains to be verified whether the convergence to a trivial steady state is true for all periodic problems. 

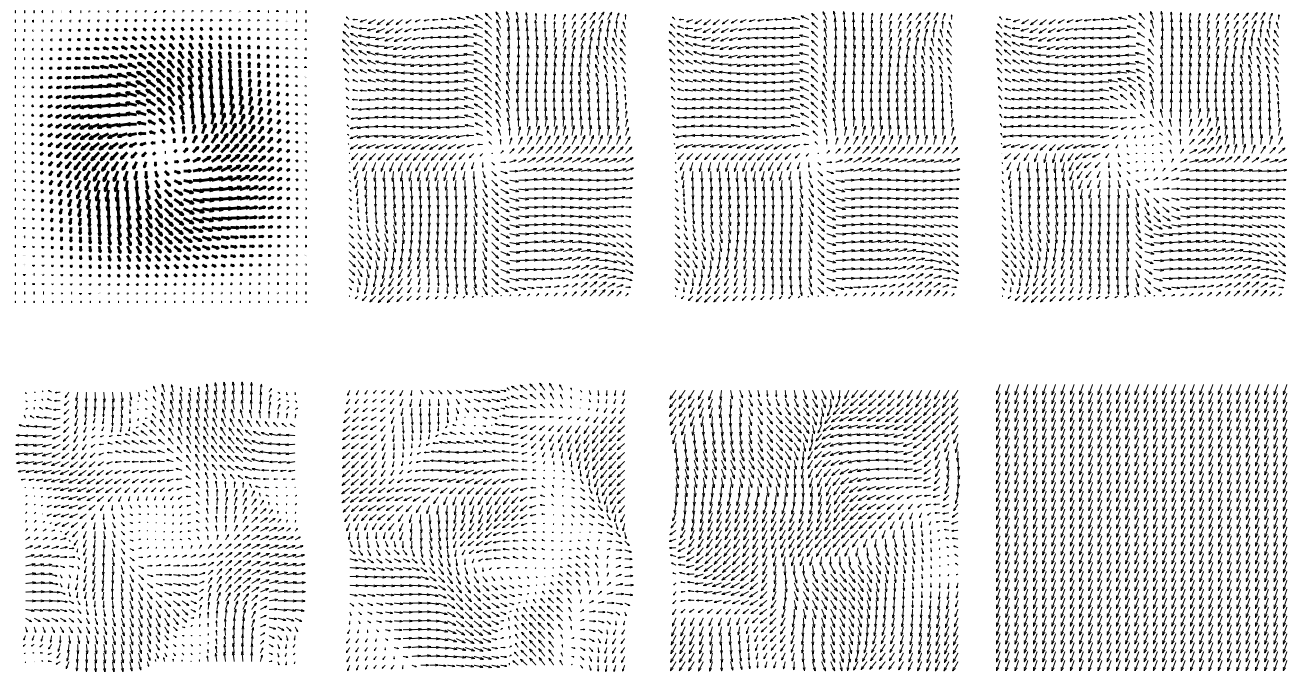

FIG. 5.1. Vector plots of $d$ (left to right): $t=0.0,0.8,1.3,1.8$ (top), $t=2.3,2.8,3.1,5.0$ (bottom).
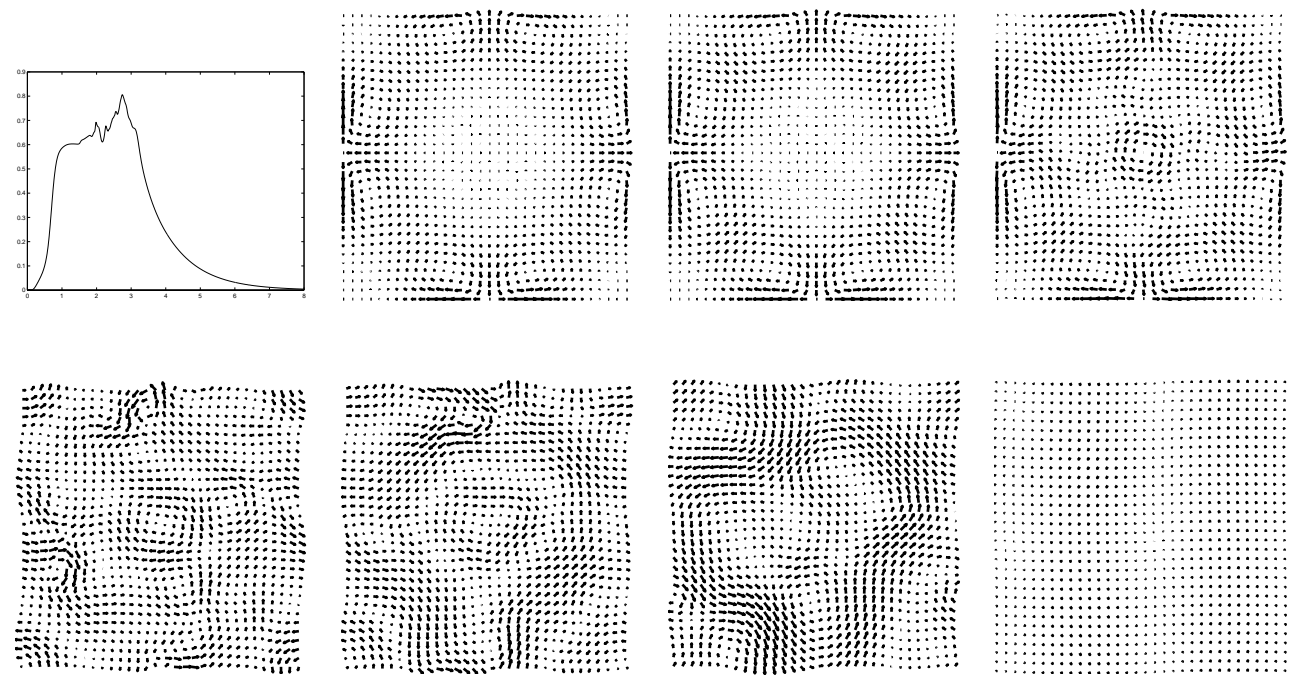

FIG. 5.2. The time evolution of $\|u\|$ (top left) and the vector plots of $u$ (left to right): $t=$ $0.8,1.3,1.8$ (top), $t=2.3,2.8,3.1,5.0$ (bottom).

5.2. Annihilation of pairs of $\pm \mathbf{1}$-degree defects. We now study the annihilation of \pm 1 -degree defects for the director field which are given in the interior of the square domain. In the first experiment, we consider two defects of opposite signs symmetrically located at $( \pm \pi / 2,0)$, i.e., the initial director field is given by

$$
d_{0}(x, y)=0.2 * \sin \left(\frac{x+\pi}{2}\right)^{2} \sin \left(\frac{y+\pi}{2}\right)^{2}\left(x^{2}+y^{2}-\frac{\pi^{2}}{4.0}, \pi y\right) .
$$

The annihilation of opposite defects can be clearly observed from Figure 5.3. The corresponding induced velocity field is plotted in Figure 5.4. This annihilation process 

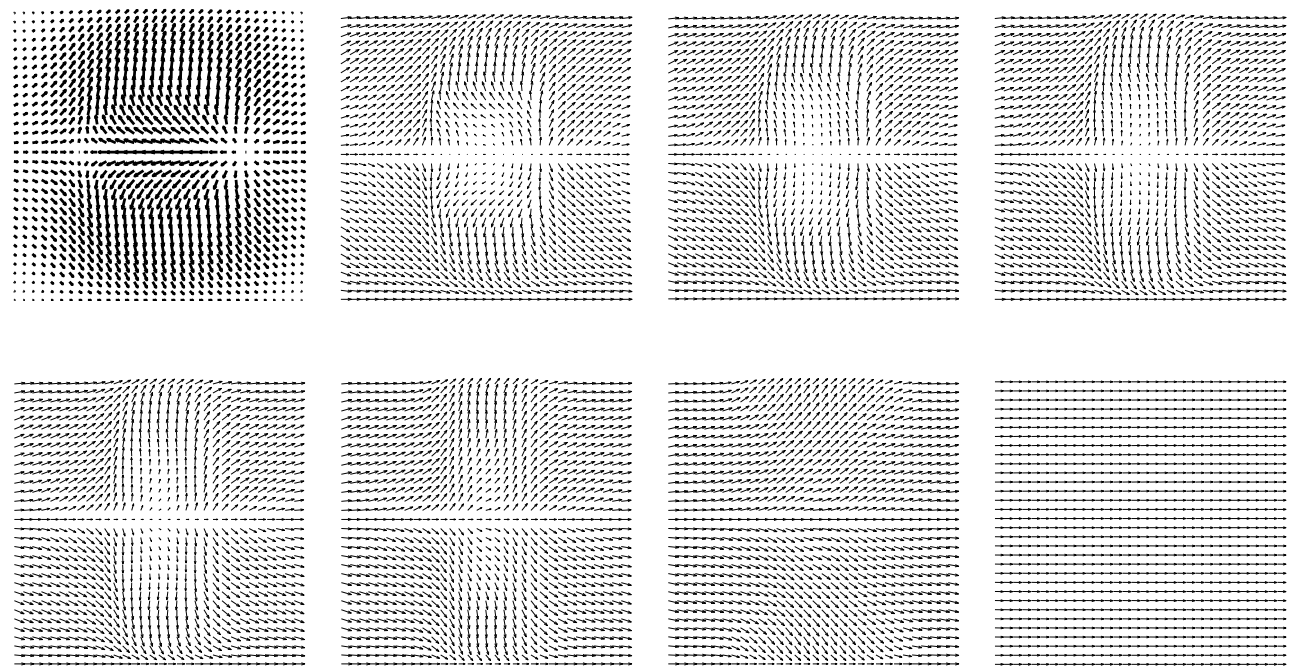

Fig. 5.3. Vector plots of $d$ (left to right): $t=0.1,0.5,0.6,0.65$ (top); $t=0.7,0.8,1.0,4.0$ (bottom).
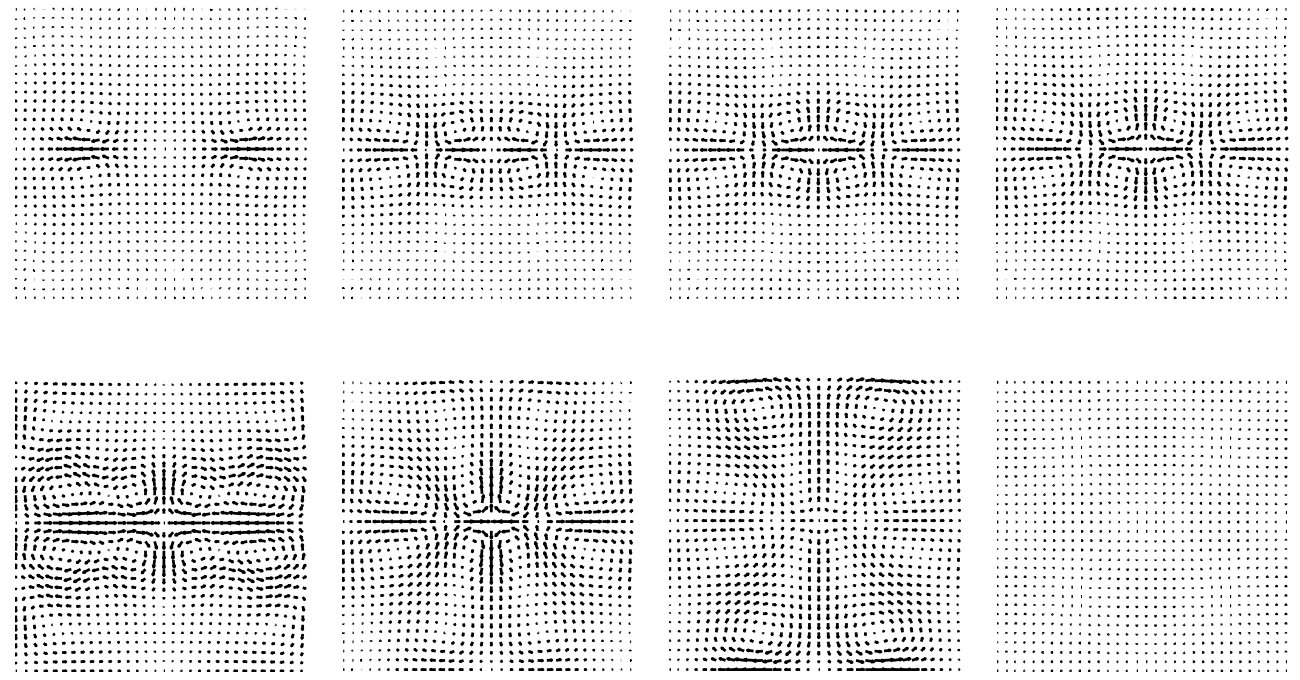

Fig. 5.4. Vector plots of $u$ (left to right): $t=0.1,0.5,0.6,0.65$ (top); $t=0.7,0.8,1.0,4.0$ (bottom).

is not greatly affected by the periodic boundary conditions.

For the next experiment, we consider two defects of the same signs symmetrically located at $( \pm \pi / 2,0)$, i.e., the initial director field is given by

$$
d_{0}(x, y)=0.2 * \sin \left(\frac{x+\pi}{2}\right)^{2} \sin \left(\frac{y+\pi}{2}\right)^{2}\left(x^{2}-y^{2}+\frac{\pi^{2}}{4.0}, 2 x y\right) .
$$

The plots of director fields and velocity fields at various times are presented in Figures 5.5 and 5.6.

The time evolution shows that, due to the periodic boundary conditions, two negative defects come in from the midside of the boundary and annihilate the defects 

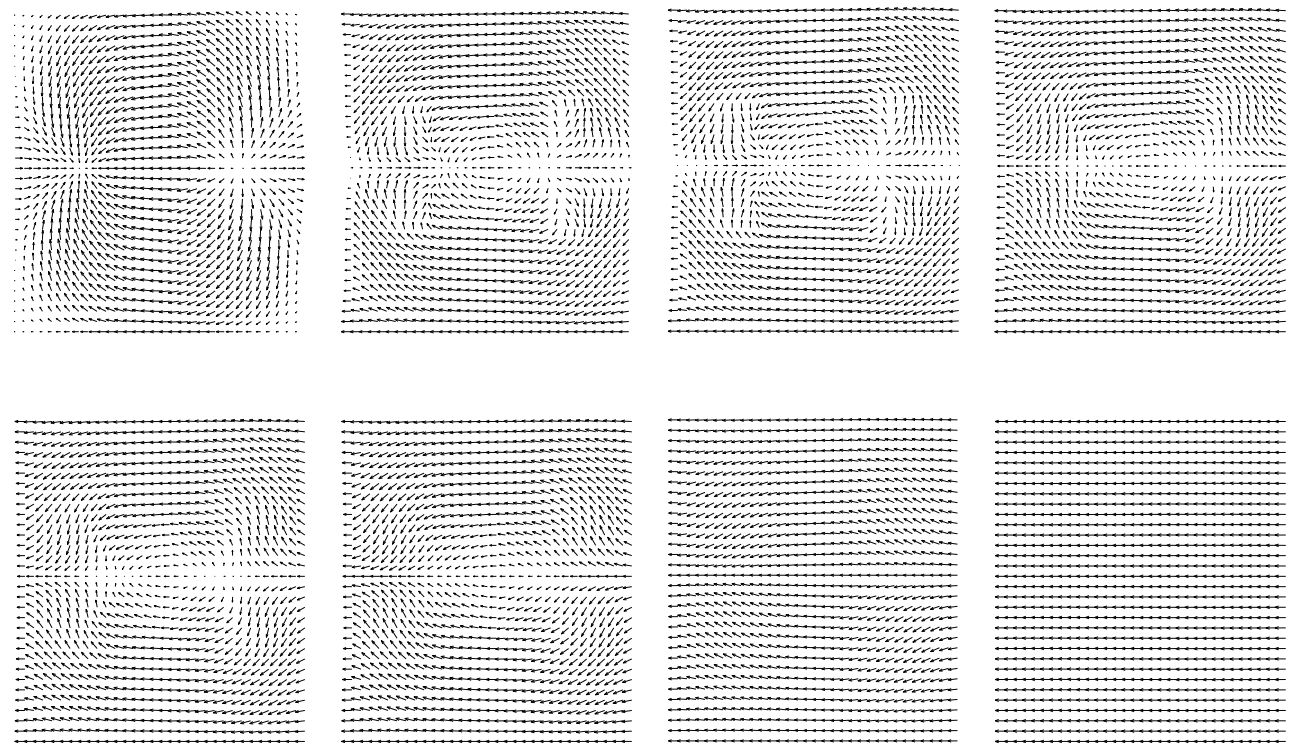

FiG. 5.5. Vector plots of $d$ (left to right): $t=0.2,0.53,0.55,0.63$ (top); $t=0.64,0.7,1.0,2.5$ (bottom).
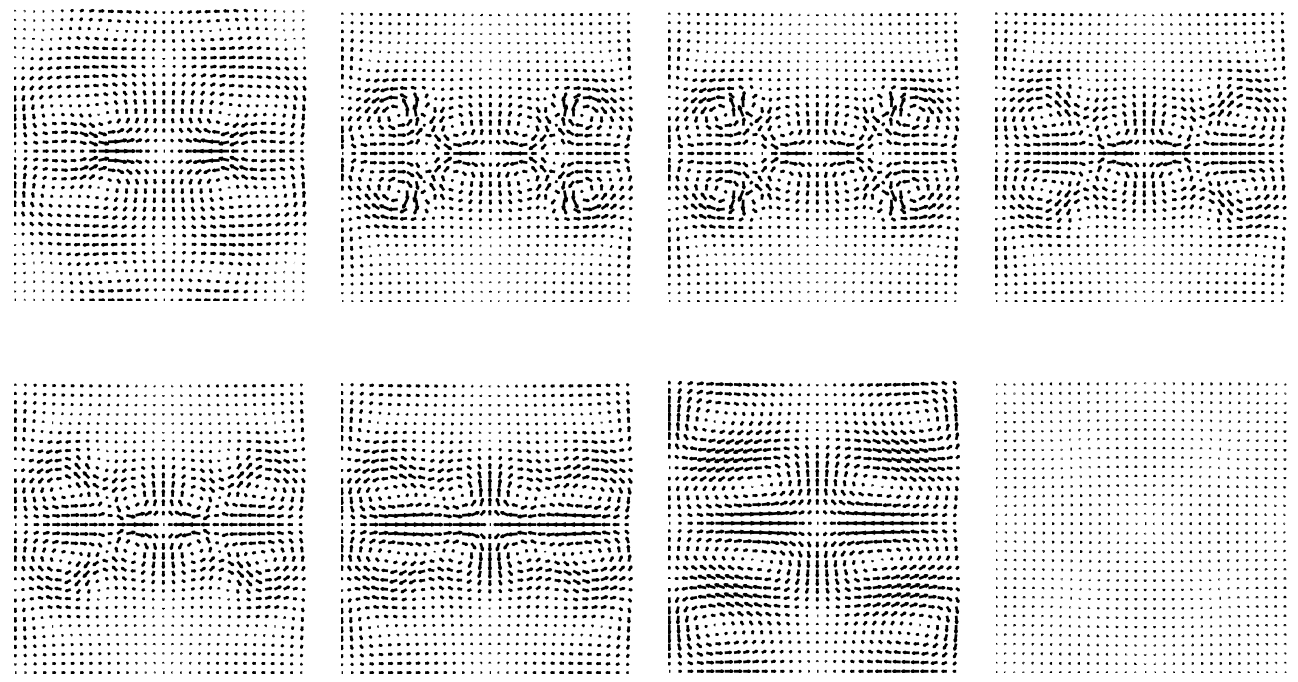

Fig. 5.6. Vector plots of $u$ (left to right): $t=0.2,0.53,0.55,0.63$ (top); $t=0.64,0.7,1.0,2.5$ (bottom).

originally present in the sample. The defect nucleation at the boundary occurs roughly around $t=0.54$ and the annihilation takes place around $t=0.63$.

Even though a time plot of the norm of the velocity field $u$ is not provided here, as pointed out earlier, the induced velocity field again approaches zero at large time and the steady states are reached in both cases. 

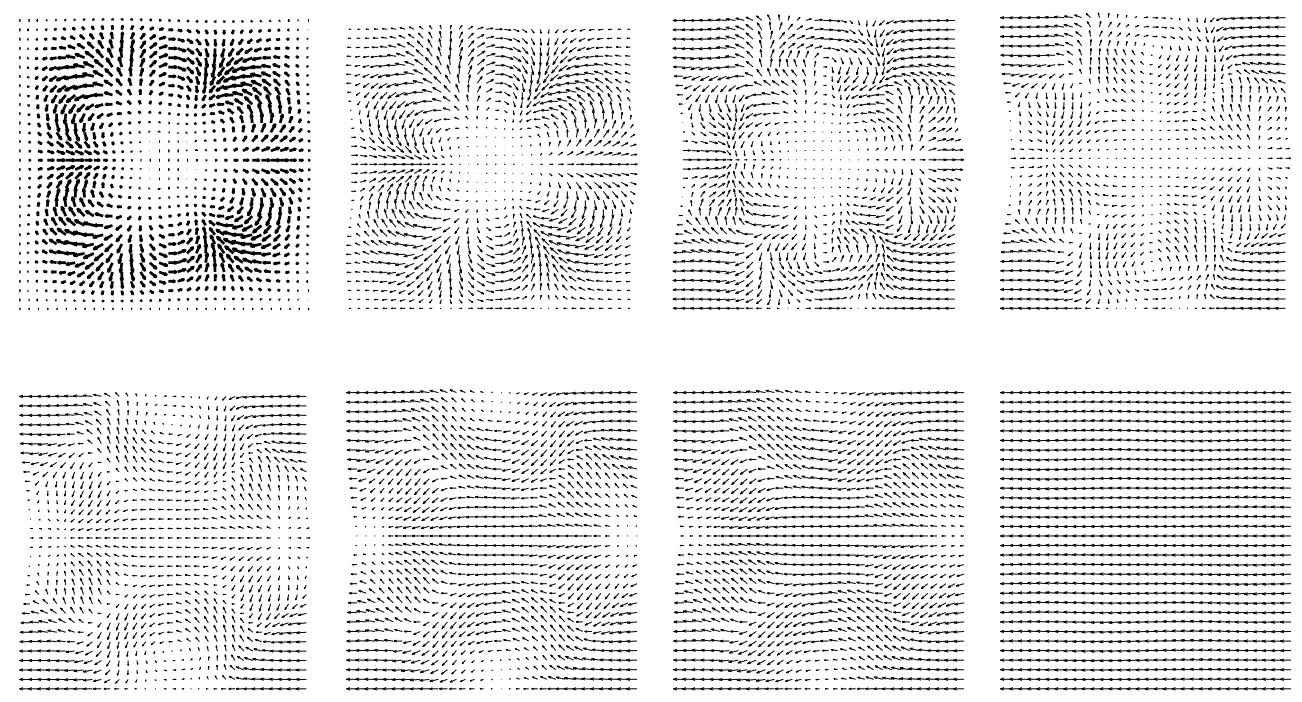

FIG. 5.7. Vector plots of $d$ (left to right): $t=0.0,0.1,0.25,0.35$ (top); $t=0.45,0.55,0.6,1.0$ (bottom).
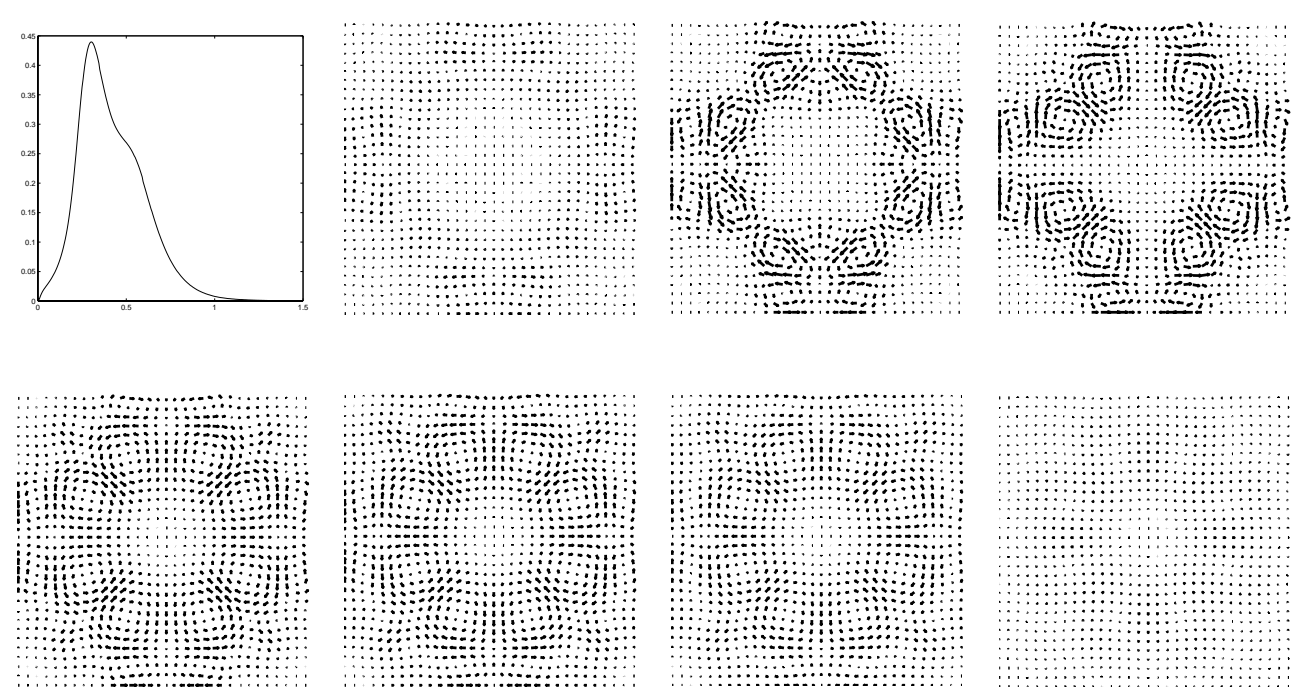

FIG. 5.8. The time evolution of $\|u\|$ (top left) and the vector plots of $u$ (left to right): $t=$ $0.1,0.25,0.35$ (top); $t=0.45,0.55,0.6,1.0$ (bottom).

5.3. Splitting of a +4-degree defect. We now study the splitting of a +4 degree defect for the director field located at the origin, i.e., the initial director field is given by

$$
d_{0}(x, y)=0.2 * \sin ((x+\pi) / 2)^{2} \sin ((y+\pi) / 2)^{2}\left(x^{4}-6 x^{2} y^{2}+y^{4}, 4 x y\left(x^{2}-y^{2}\right)\right) .
$$

The plots of director fields and velocity fields at various times and the time evolution of $\|u\|$ are presented in Figures 5.7 and 5.8.

As time increases, the +4 -degree defect quickly splits into four +1-degree defects 
and they in turn annihilate with the opposite defects coming in from the bound-

ary. Again, the initial splitting of high degree defects is not greatly affected by the periodicity, though the latter annihilation process is.

\section{REFERENCES}

[1] F. Alouges, A new algorithm for computing liquid crystal stable configurations: The harmonic mapping case, SIAM J. Numer. Anal., 34 (1997), pp. 1708-1726.

[2] C.V. Brown, M.J. Towler, V.C. Hui, and G.P.A. Bryan-Brown, Numerical analysis of nematic liquid crystal alignment on asymmetric surface grating structures, Liquid Crystals, 27 (2000), pp. 233-242.

[3] C. Canuto, M.Y. Hussaini, A. Quarteroni, and T.A. Zang, Spectral Methods in Fluid Dynamics, Springer-Verlag, Berlin, 1988.

[4] L. Chen And J. Shen, Applications of semi-implicit Fourier-spectral method to phase-field equations, Comput. Phys. Comm., 108 (1998), pp. 147-158.

[5] S. Chono, T. Tsuji, And M.M. Denn, Spatial development of director orientation of tumbling nematic liquid crystals in pressure-driven channel flow, J. Non-Newtonian Fluid Mech., 79 (1998), pp. 515-527.

[6] J.B. Davies, S. Day, F. DiPasquale, and F.A. Fernandez, Finite-element modelling in 2-D of nematic liquid crystal structures, Electron. Lett., 32 (1996), pp. 582-583.

[7] T.A. Davis and E.C. Gartland JR., Finite element analysis of the Landau-de Gennes minimization problem for liquid crystals, SIAM J. Numer. Anal., 35 (1998), pp. 336-362.

[8] C. Denniston, E. Orlandini, and J. Yeonmans, Simulations of liquid crystal hydrodynamics, Europhys. Lett., 52 (2000), pp. 481-487.

[9] J. ERICKson, Conservation laws for liquid crystals, Trans. Soc. Rheol., 5 (1961), pp. $22-34$

[10] J. ERICKson, Equilibrium theory of liquid crystals, in Advances in Liquid Crystals, Vol. 2, G. Brown, ed., Academic Press, New York, 1976, pp. 233-398.

[11] M.G. Forest, Q. WANG, AND H. ZHOU,, Homogeneous pattern selection and director instabilities of nematic liquid crystal polymers induced by elongational flows, Phys. Fluids, 12 (2000), pp. 490-498.

[12] B.-Y. Guo, A class of difference schemes of two-dimensional viscous fluid flow, Acta Math. Sinica, 17 (1974), pp. 242-258.

[13] B.-Y. Guo, Difference Methods for Partial Differential Equations, Science Press, Beijing, 1988.

[14] B.-Y. Guo,, Spectral Methods and Their Applications, World Scientific, Singapore, 1998.

[15] M. Inoue, H. Moritake, And K. Toda, Periodic property of domain in nematic liquid crystal induced by elastic wave, Japanese J. Appl. Phys. I, 39 (2000), pp. 3125-3129.

[16] F. John, Partial Differential Equations, 4th ed., Springer-Verlag, New York, 1982.

[17] O.A. Ladyzhenskaya, The Mathematical Theory of Viscous Incomprehensible Flow, 2nd ed., Gordon and Breach, New York, 1969 (in English).

[18] F.M. LesLIE, Theory of flow phenomena in liquid crystals, in Advances in Liquid Crystals, Vol. 4, G. Brown, ed., Academic Press, New York, 1979, pp. 1-81.

[19] F.H. LiN, Nonlinear theory of defects in nematic liquid crystals: Phase transition and flow phenomena, Comm. Pure. Appl. Math., Vol. 42 (1989), pp. 789-914.

[20] F.H. Lin, Mathematics theory of liquid crystals, in Applied Mathematics at the Turn of Century, to appear.

[21] F.H. LiN, private communication, Courant Institute, New York, 2000

[22] F.H. Lin AND C. LiU, Nonparabolic dissipative systems modeling the flow of liquid crystals, Comm. Pure. Appl. Math., Vol. 48 (1995), pp. 501-537.

[23] J.L. Lions, Quelques Méthodes de Résolution des Problèmes aux Limites Non Linéaires, Dunod, Paris, 1969.

[24] C. Liu and N.J. Walkington, Approximation of liquid crystals flows, SIAM J. Numer. Anal., 37 (2000), pp. 725-741.

[25] K. Migler And R.B. Meyer, Fluid-flow-induced pattern-formation in liquid-crystals in a rotating magnetic-field, Phys. Rev. E (3), 48 (1993), pp. 1218-1227.

[26] V. Mocella, C. Ferrero, M. Iovane, and R. Barberi, Numerical investigation of surface distortion and order parameter variation in nematics, Liquid Crystals, 26 (1999), pp. $1345-1350$.

[27] M. Reichenstein, T. Seitz, And H. Trebin, Numerical simulations of three dimensional liquid crystal cells, Molecular Crystals and Liquid Crystal Sci. and Tech., 330 (1999), p. 793.

[28] J. Shen, Efficient spectral-Galerkin method I. Direct solvers of second-and fourth-order equations using Legendre polynomials, SIAM J. Sci. Comput., 15 (1994), pp. 1489-1505. 
[29] J. Shen, Efficient spectral-Galerkin method II. Direct solvers of second-and fourth-order equations using Chebyshev polynomials, SIAM J. Sci. Comput., 16 (1995), pp. 74-87.

[30] R. Temam, Navier-Stokes Equations, North-Holland, Amsterdam, 1977.

[31] J. Zhu, L. Chen, J. Shen, And V. Tikare, Coarsening kinetics from a variable mobility. Cahn-Hilliard equation-application of semi-implicit Fourier spectral method, Phys. Rev. E (3), 60 (1999), pp. 3564-3572. 